Excited Spectator Electron Effects on Spectral Line Shapes

C. A. Iglesias

October 15, 2009

High Energy Density Physics 
This document was prepared as an account of work sponsored by an agency of the United States government. Neither the United States government nor Lawrence Livermore National Security, LLC, nor any of their employees makes any warranty, expressed or implied, or assumes any legal liability or responsibility for the accuracy, completeness, or usefulness of any information, apparatus, product, or process disclosed, or represents that its use would not infringe privately owned rights. Reference herein to any specific commercial product, process, or service by trade name, trademark, manufacturer, or otherwise does not necessarily constitute or imply its endorsement, recommendation, or favoring by the United States government or Lawrence Livermore National Security, LLC. The views and opinions of authors expressed herein do not necessarily state or reflect those of the United States government or Lawrence Livermore National Security, LLC, and shall not be used for advertising or product endorsement purposes. 


\title{
Excited Spectator Electron Effects on Spectral Line Shapes
}

\author{
CARlos A. Iglesias \\ Lawrence Livermore National Laboratories \\ P.O. Box 808, Livermore, CA 94550, USA
}

\begin{abstract}
Excited spectator electron effects on Stark broadened spectral line shapes of transitions involving tightly bound electrons are investigated. It is shown that the interference terms in the electron impact broadening are essential to describe the overlapping lines generated by these configurations (e.g.; dielectronic satellite lines). The main impact is narrower spectral features and reduced far wing intensities compared to calculations neglecting the interference terms.
\end{abstract}




\section{Introduction}

It is known that theoretical Rosseland mean opacities [1] can be sensitive to line shape models. [2] For example, low photon absorption regions have the largest impact on the Rosseland mean opacity; thus, line wings can be important. Another instance is astrophysical opacities where line clusters from heavier elements can form either broad absorption features or a picket fence of sharp resonances depending on the line widths. The former can appreciably increase the opacity while the latter has little impact on the results. Thus, the entire line shape can influence opacity calculations. Due to computational constraints, however, it is impractical for opacity models to incorporate complex line shape theories. Therefore, simplified formulas that presumedly capture the essence of the relevant processes are employed.

An unresolved issue is the role of spectator electrons on line profiles. Specifically, opacity calculations require line shapes for radiative transitions involving tightly bound electrons in configurations with excited spectator electrons. In these cases, the level width contribution from an excited spectator electron, which increases as $n^{4}$ with $n$ the spectator electron principal quantum number, [3] can dominate the line profile. On the other hand, it is expected that the spectator electron contribution to the line shape should diminish as its coupling to the optical electron becomes weaker as $n$ increases.

The two apparently contradictory statements above can be reconciled with a more complete treatment of line broadening that includes the interference terms. [3,4] These terms are often neglected since they are small for plasma diagnostic lines emphasized by most research efforts. $[3,5]$ One exception is radio frequency lines where the interference terms are known to reduce the line widths. [3,6,7] Another exception, more closely related to the present problem, is Sobelman [7,8] who discussed line narrowing by interference effects for relaxation processes only affecting states weakly coupled to the radiating electron.

The discussion begins with a brief review of Stark broadening theory. Examples are then provided showing how the interference terms can lead to narrow spectral lines even though there are large level widths from weakly coupled spectator electrons. The examples are simple and yield analytical expressions that provide insight to the interference effects. This is followed by a more general discussion of interference effects on the far line wing. Finally, calculations for dielectronic satellite lines of Al IV demonstrate the impact of the interference terms that were previously neglected in plasma characterization using such lines. 


\section{Stark broadening theory}

The spectral power emission or absorption of a quantum-mechanical system can be written in terms of a line shape function. [3] For Stark broadening the number of radiators is assumed small relative to the number of perturbers so that the radiation from different emitters adds incoherently. The line shape function is then approximated by that of a single radiator in a bath of electrons and quasi-static ions, which for dipole transitions can be written in terms of "line space" elements as (neglecting Doppler broadening) [3,4]

$$
\begin{aligned}
I(\omega) & =\pi^{-1} \operatorname{Re} \int_{o}^{\infty} d t e^{i \omega t} \operatorname{Tr}\left\{\vec{d} \cdot e^{-i L t / \hbar} \rho \vec{d}\right\} \\
& \approx-\pi^{-1} \operatorname{Im} \int_{o}^{\infty} d F W(F) \sum_{\mu v} \sum_{\mu^{\prime} v^{\prime}} \vec{d}_{\mu v}^{*} \cdot R_{\mu v, \mu^{\prime} v^{\prime}}(\omega ; F) \rho_{\mu^{\prime}} \vec{d}_{\mu^{\prime} v^{\prime}}
\end{aligned}
$$

where $L$ is the Liouville operator defined by commutation with the total Hamiltonian of the system. Note that the second line of Eq. (2.1) assumed the density matrix is stationary (i.e., $L \rho=0$ ) and the trace was preformed with isolated radiator internal states. Accordingly, a line element $\mu v$ comprises $\mu^{\prime} s$ and $v^{\prime} s$ that are, respectively, initial and final $|J M\rangle$ atomic states with total angular momentum $J$ and magnetic number $M$. Also, $\rho_{\mu}$ is the population of state $\mu$, $\vec{d}_{\mu v}$ are matrix elements of the radiator dipole operator with * denoting the complex conjugate, and $W(F)$ is the probability distribution of the quasi-static Stark field, $\vec{F}$. The resolvent, which describes the time evolution of the radiator dipole operator, is defined by its inverse

$$
R_{\mu v, \mu^{\prime} v^{\prime}}^{-1}(\omega ; F)=\Delta \omega_{\mu v} \delta_{\mu^{\prime} \mu} \delta_{v^{\prime} v}-\left(\vec{d}_{\mu \mu^{\prime}} \delta_{v v^{\prime}}-\delta_{\mu \mu^{\prime}} \vec{d}_{v v^{\prime}}^{*}\right) \cdot \vec{F} / \hbar-H_{\mu v, \mu^{\prime} v^{\prime}}(\omega)
$$

with detuning frequency

$$
\Delta \omega_{\mu v}=\omega-\omega_{\mu}+\omega_{v}
$$

and $\hbar \omega_{\alpha}$ the energy of radiator internal state $|\alpha\rangle$. The "width and shift" operator, $H(\omega)$, contains the plasma electron contribution to the relaxation processes of the radiator states. [9-14] In principle, the sum in Eq. (2.1) is over a complete set of states involving not only discreet lines but also bremsstrahlung and photon recombination spectra. In applications, however, the radiator levels are restricted to those whose elements of $\vec{d}$ and $R(\omega ; F)$ significantly contribute to transitions in the spectral range of interest. 
The expression in line space (also Liouville or double atom representation) is more complex than the usual Hamiltonian notation. It has a direct appeal, however, since experimentalists observe lines not energy levels. That is, in the absence of the plasma, $R^{-1}(\omega) \rightarrow R_{O}^{-1}(\omega)$ whose eigenvalues are the resonance frequencies $\Delta \omega$ in Eq. (2.3). The effect of the plasma is to introduce a perturbation to $R_{o}(\omega)$. The plasma electrons produce non-Hermitian widths and shifts while the quasi-static ions lead to line shifts to be averaged over the field distribution.

\subsection{Dipole matrix elements}

The dipole matrix elements are obtained using the Wigner-Eckart theorem, [15]

$$
\vec{d}_{\mu v}=\left\langle J_{\mu} M_{u}|\vec{d}| J_{v} M_{v}\right\rangle=e a_{o}(-1)^{J_{\mu}-M_{\mu}}\left(\begin{array}{ccc}
J_{\mu} & 1 & J_{v} \\
-M_{\mu} & M_{\mu}-M_{v} & M_{v}
\end{array}\right) S_{\mu v}^{1 / 2}
$$

with $S_{\mu \nu}^{1 / 2}$ the reduced matrix element in units of the Bohr radius $a_{o}$ (see Appendix A). The scalar product yields,

$$
\begin{aligned}
& \vec{d}_{\mu v}^{*} \cdot \vec{d}_{\mu^{\prime} v^{\prime}}=e^{2} a_{O}^{2} \delta_{M_{\mu}-M_{v}, M_{\mu^{\prime}}-M_{v^{\prime}}}(-1)^{J_{\mu}-M_{\mu}+J_{\mu^{\prime}}-M_{\mu^{\prime}}} \\
& \times\left(\begin{array}{ccc}
J_{\mu} & 1 & J_{v} \\
-M_{\mu} & M_{\mu}-M_{v} & M_{v}
\end{array}\right)\left(\begin{array}{ccc}
J_{\mu^{\prime}} & 1 & J_{v^{\prime}} \\
-M_{\mu^{\prime}} & M_{\mu^{\prime}}-M_{v^{\prime}} & M_{v^{\prime}}
\end{array}\right) S_{\mu v}^{1 / 2} S_{\mu^{\prime} v^{\prime}}^{1 / 2}
\end{aligned}
$$

where the symmetry relations of the $3-\mathrm{j}$ symbols and reduced matrix elements were used to manipulate the result. [15]

\subsection{Second-order theory and dipole approximation}

The tetradic or double atom representation of $H(\omega)$ is obtained by taking matrix elements of the product, [9-14]

$$
\langle\mu|H(\omega) Y| v\rangle=\sum_{\mu^{\prime} v^{\prime}} H_{\mu v, \mu^{\prime} v^{\prime}}(\omega) Y_{\mu^{\prime} v^{\prime}}
$$

where $Y$ is an arbitrary operator in the radiator subspace. The width contributions from electron collisions can be written in the form

$$
\operatorname{Im} H_{\mu v, \mu^{\prime} v^{\prime}}(\omega)=\delta_{\mu^{\prime} \mu} \delta_{v^{\prime} v} \gamma_{\mu v}(\omega)-\left(1-\delta_{\mu^{\prime} \mu} \delta_{v^{\prime} v}\right) \tau_{\mu v, \mu^{\prime} v^{\prime}}(\omega)
$$

Here, $\gamma_{\mu \nu}$ are the level widths given by the diagonal elements of $H(\omega)$ and the interference terms, $\tau_{\mu v, \mu^{\prime} v^{\prime}}$, refer to the off-diagonal elements of $H(\omega)$ that can be important for overlapping lines but are negligible for isolated lines. [3,4] Although there are coherent terms in the elastic 
scattering contributions to $\gamma_{\mu \nu}$ that can affect both isolated and overlapping lines, [16] it is the effect of $\tau_{\mu v, \mu^{\prime} v^{\prime}}$ on overlapping lines that is the subject of interest here.

In second-order theory and dipole approximation discussed in Appendix B, the level widths are independent of magnetic quantum numbers (neglecting detailed balance [14]),

$$
\gamma_{\mu \nu}^{(2)}=\sum_{J_{\sigma}}\left\{\frac{\left|S_{\mu \sigma}^{1 / 2}\right|^{2}}{2 J_{\mu}+1} g\left(\Delta \omega_{\sigma v}\right)+\frac{\left|S_{v \sigma}^{1 / 2}\right|^{2}}{2 J_{v}+1} g\left(\Delta \omega_{\mu \sigma}\right)\right\}
$$

where the sum is over total angular momentum levels of the isolated radiator. The function $g(\omega)$ involves a thermal average over the collision momentum transfer,

$$
g(\omega)=\frac{4 e^{4} a_{o}^{2}}{3 \hbar^{2}} \int_{o}^{k_{m}} d k S(k, \omega)
$$

with $S(k, \omega)$ the electron gas dynamic structure factor and $k_{m}$ keeps the integrals finite.

In the same approximations the interference terms are given by

$$
\begin{aligned}
& \tau_{\mu v, \mu^{\prime} v^{\prime}}^{(2)}=\tau_{\mu^{\prime} v^{\prime}, \mu v}^{(2)}=\delta_{M_{\mu^{\prime}}-M_{\mu}, M_{v^{\prime}}-M_{v}}(-1)^{J_{\mu}-M_{\mu}}\left(\begin{array}{ccc}
J_{\mu} & 1 & J_{\mu^{\prime}} \\
-M_{\mu} & M_{\mu}-M_{\mu^{\prime}} & M_{\mu^{\prime}}
\end{array}\right) S_{\mu \mu^{\prime}}^{1 / 2} \\
& \times(-1)^{J_{v}-M_{v}}\left(\begin{array}{ccc}
J_{v} & 1 & J_{v^{\prime}} \\
-M_{v} & M_{v}-M_{v^{\prime}} & M_{v^{\prime}}
\end{array}\right) S_{v v^{\prime}}^{1 / 2}\left\{g\left(\Delta \omega_{\mu^{\prime} v}\right)+g\left(\Delta \omega_{\mu v^{\prime}}\right)\right\}
\end{aligned}
$$

The properties of the 3-j symbols, reduced dipole matrix elements, and the terms in brackets lead to the symmetric results. It follows that the interference terms can entangle the initial and final level manifolds even in cases where their coupling through electron collisional excitations and the quasi-static Stark fields are neglected.

\subsection{Line shape calculation}

The evaluation of the line shape requires radiator energy levels and reduced dipole matrix elements usually obtained from atomic data calculations. [17-19] With these data, together with the description for electron collisional excitations, the matrices above are constructed. The calculation requires a matrix inversion (or equivalent) for each frequency and Stark field; however, in some cases approximations can accelerate this step. [18] Also required is the microfield distribution to average over the quasi-static fields. [20,21] Often thermal equilibrium is assumed, but non-thermal equilibrium radiator state populations are also of interest. [19] 
Finally, line shape calculations for multi-electron ions involve significant computational difficulties and the interference terms considerably increase the size of the matrices. Consequently, the interference terms are often neglected for practical reasons rather than systematic analysis.

\section{Spectator electron relaxation: $L S$ coupling}

To study the interference effects, a two-electron ion with level structure displayed in Fig. 1 is considered. The configuration term structure assumes $L S$ coupling and only the singlet states (total spin $S=0$ ) are included. The triplet system (total spin $S=1$ ) is independent and considered separately below. Although not necessary, the $L S$-term structure of the $2 p n p$ configuration is assumed degenerate simplifying the algebra. The radiative transitions of interest connect the $1 s$ and $2 p$ orbitals.

The relaxation of the system is entirely due to plasma electron collisions with the excited spectator electron in the $n s$ or $n p$ subshell. Thus, the line broadening depends on the coupling between the optical and spectator electron. Without this coupling the line shape should reduce to a $\delta$-function (natural and autoionization widths are neglected). Furthermore, the value of $n$ is assumed sufficiently large that the energy splitting of the lines is small compared to their energy (i.e.; $\delta<\omega_{o}$ in Fig. 1). Consequently, $g\left(\Delta \omega_{\mu v}\right) \approx g(\Delta \omega)$ with $\Delta \omega=\omega-\omega_{o}$ for all level widths and interference terms. Finally, the quasi-static Stark fields are neglected.

\subsection{Diagonal blocks}

The line shape calculation in this example involves 48 line elements. The resolvent operator has block diagonal form where only 3 similar $(7 \times 7)$ blocks contribute to the profile; all others have vanishing dipole transitions. The block with $\Delta m_{\mu v}=m_{\mu}-m_{v}=0$ involves the line elements,

$$
\{\mu v\}=3_{o} 1_{o}, 4_{o} 2_{o}, 5_{+} 2_{+}, 5_{-} 2_{-}, 6_{+} 2_{+}, 6_{o} 2_{o} \text {, and 6_2 }
$$

where the labels are the levels in Fig. 1 with magnetic quantum numbers as subscripts. The blocks with $\Delta m_{\mu \nu}= \pm 1$ yield identical contributions to the profle so are not explicitly discussed.

\subsection{Reduced dipole matrix elements}

The electron collisional excitations involve only levels within either the upper or lower manifold; that is, no collisional excitations are allowed between initial and final levels. The $L S$ 
reduced dipole matrix elements connecting the levels in Fig. 1 necessary for the widths are obtained using formulas in Appendix C,

$$
\begin{aligned}
& S_{21}^{1 / 2}=-S_{12}^{1 / 2}=S_{35}^{1 / 2}=S_{53}^{1 / 2}=1, \\
& S_{43}^{1 / 2}=-S_{34}^{1 / 2}=1 / \sqrt{3} \\
& S_{63}^{1 / 2}=-S_{36}^{1 / 2}=\sqrt{5 / 3}
\end{aligned}
$$

The results are in units of $e a_{o} R_{n s, n p}$ where the spectator electron radial wavefunction dependence on the optical electron was neglected.

The radiative transitions connect only levels in the upper manifold to levels in the lower manifold. Again using $L S$ formulas in Appendix C

$$
\begin{aligned}
& S_{31}^{1 / 2}=-S_{13}^{1 / 2}=-S_{25}^{1 / 2}=-S_{52}^{1 / 2}=1, \\
& S_{42}^{1 / 2}=-S_{24}^{1 / 2}=1 / \sqrt{3}, \\
& S_{62}^{1 / 2}=-S_{26}^{1 / 2}=\sqrt{5 / 3}
\end{aligned}
$$

The results are in units of $e a_{o} R_{1 s, 2 p}$ and the optical electron radial wavefunction dependence on the spectator electron was neglected. All other reduced dipole matrix elements are assumed to vanish.

\subsection{Line width and shift}

Straightforward substitution of the results in Section 3.2 yields for the level widths in Eq. (2.2.3), (the second-order superscript is dropped for brevity)

$$
\begin{aligned}
& \gamma_{31}=3 \gamma_{o}, \\
& \gamma_{42}=\gamma_{52}=\gamma_{62}=\gamma_{o}
\end{aligned}
$$

where the subscript correspond to spectral lines connecting the levels in Fig. 1 and $\gamma_{o}$ is defined by the impact limit,

$$
\gamma_{o}=\frac{2}{3} R_{n s, n p}^{2} g(\Delta \omega \rightarrow 0)
$$

that makes it independent of frequency. 
The non-vanishing interference terms for the $\Delta m=0$ block from Eq. (2.2.5) all involve the line element $3{ }_{o} 1_{o}$,

$$
\begin{aligned}
& \tau_{3_{o} 1_{o}, 4_{o} 2_{o}}=-\gamma_{o} / \sqrt{3} \\
& \tau_{3_{o} 1_{o}, 6_{o} 2_{o}}=\gamma_{o} \sqrt{2 / 3} \\
& \tau_{3_{o} 1_{o}, 5_{-} 2_{-}}=-\tau_{3_{o} 1_{o}, 5_{+} 2_{+}}=\tau_{3_{o} 1_{o}, 6_{+} 2_{+}}=\tau_{3_{o} 1_{o}, 6_{-} 2_{-}}=\gamma_{o} / \sqrt{2}
\end{aligned}
$$

Symmetry of $\tau_{\mu v, \mu^{\prime} v^{\prime}}$ completes the matrix elements required for this block.

The dispersion relation provides the second-order line shifts (see Appendix B). Assuming a frequency independent width then yields

$$
\begin{aligned}
\operatorname{Re} M^{(2)}(\omega) & =P \int_{-\infty}^{\infty} \frac{d \omega^{\prime}}{\pi} \frac{\operatorname{Im} M^{(2)}\left(\omega^{\prime}\right)}{\omega-\omega^{\prime}} \\
& \propto \frac{\gamma_{O}}{\pi} P \int_{-\infty}^{\infty} d \omega^{\prime} \frac{1}{\omega-\omega^{\prime}}=0
\end{aligned}
$$

where $P P$ denotes the Cauchy principal part. For simplicity, shifts due to the average radiatorplasma interaction $[3,11]$ are neglected.

\subsection{Dipole line strength}

The dot product of the dipole matrix elements for the radiative transition in the $\Delta m=0$ block and basis set in Eq. (3.1.1) is given by

$$
\frac{\vec{d}_{\mu v}^{*} \cdot \vec{d}_{\mu^{\prime} v^{\prime}}}{e^{2} a_{o}^{2} R_{1 s, 2 p}^{2}}=\frac{1}{6}\left[\begin{array}{ccccccc}
2 & -2 / \sqrt{3} & -\sqrt{2} & \sqrt{2} & \sqrt{2} & 2 \sqrt{2 / 3} & \sqrt{2} \\
2 / 3 & \sqrt{2 / 3} & -\sqrt{2 / 3} & -\sqrt{2 / 3} & -2 \sqrt{2} / 3 & -\sqrt{2 / 3} \\
& 1 & -1 & -1 & -2 / \sqrt{3} & -1 \\
& & & 1 & -1 & 2 / \sqrt{3} & 1 \\
& & & 1 & 2 / \sqrt{3} & 1 \\
& & & & 4 / 3 & 2 / \sqrt{3} \\
& & & & & 1
\end{array}\right]
$$

and the matrix is symmetric so the lower triangle is left blank for brevity.

\subsection{Resolvent operator}

The $\Delta m=0$ block of $R^{-1}$ in the basis set of Eq. (3.1.1) is the symmetric matrix 


$$
\frac{R_{\mu v, \mu^{\prime} v^{\prime}}^{-1}}{\delta}=\left[\begin{array}{ccccccc}
A & i \Gamma / \sqrt{3} & i \Gamma / \sqrt{2} & -i \Gamma / \sqrt{2} & -i \Gamma / \sqrt{2} & -i \Gamma \sqrt{2 / 3} & -i \Gamma / \sqrt{2} \\
& B & 0 & 0 & 0 & 0 & 0 \\
& & B & 0 & 0 & 0 & 0 \\
& & & B & 0 & 0 & 0 \\
& & & & B & 0 & 0 \\
& & & & & B & 0 \\
& & & & & & B
\end{array}\right]
$$

Again for brevity the lower triangle is left blank and the arguments of $A$ and $B$ were suppressed,

$$
\begin{aligned}
& A(x, \Gamma)=x+1+3 i \Gamma \\
& B(x, \Gamma)=x-1+i \Gamma
\end{aligned}
$$

where the variables

$$
\begin{gathered}
x=\Delta \omega / \delta \\
\Gamma=\gamma_{o} / \delta
\end{gathered}
$$

were introduced scaling the problem to the line separation frequency.

\subsection{Line shape function}

The line shape function in Eq. (2.1) can now be obtained. Inverting the matrix in Eq. (3.5.1), multiplying the results by the matrix in Eq. (3.4.1) followed by the trace, then assuming all initial states have the same occupation ( $\rho_{\mu}=\rho_{o}$ for all $\mu$ ), yields identical contributions from the three (7x7) diagonal blocks of $R$. The resulting line shape function is given by

$$
I(x)=\frac{3 \Gamma / \pi}{\left(x^{2}-1\right)^{2}+4 \Gamma^{2}(2 x-1)^{2}}
$$

where $I(x)$ was normalized by the total line strength

$$
\sum_{\mu \nu} \rho_{\mu} \vec{d}_{\mu \nu} \cdot \vec{d}_{v \mu}=4 \rho_{o} e^{2} a_{o}^{2} R_{1 s, 2 p}^{2}
$$

so that

$$
\int_{-\infty}^{\infty} d x I(x)=1
$$

The line shape without interference terms sets $\tau=0$ for all line elements yielding 


$$
I_{o}(x)=\frac{3 \Gamma}{4 \pi}\left\{\frac{1}{(x+1)^{2}+9 \Gamma^{2}}+\frac{1}{(x-1)^{2}+\Gamma^{2}}\right\}
$$

that is also normalized by the total line strength. The line shape $I_{o}(x)$ is given by the sum of two Lorentz profiles characterized by the sum of level widths for initial and final states.

\subsection{Analytical Limits}

Considering various limits helps display some features of the line shape functions above. In the far wing,

$$
\begin{aligned}
& \operatorname{Lim}_{x \rightarrow \infty} I(x)=\frac{3 \Gamma}{\pi x^{4}}\left\{1+\frac{2-16 \Gamma^{2}}{x^{2}}+\frac{16 \Gamma^{2}}{x^{3}}+O\left(\frac{1}{x^{4}}\right)\right\} \\
& \operatorname{Lim}_{x \rightarrow \infty} I_{o}(x)=\frac{3 \Gamma}{2 \pi x^{2}}\left\{1+\frac{3-5 \Gamma^{2}}{x^{2}}+\frac{16 \Gamma^{2}}{x^{3}}+O\left(\frac{1}{x^{4}}\right)\right\}
\end{aligned}
$$

Thus, the interference terms lead to a reduced line wing independent of the value of $\Gamma$. The vanishing of the $x^{-2}$ term in $I(x)$ is a more general result considered in Section 6.

For small $\Gamma$, either by $\gamma_{o} \ll<1$ or $\delta>>1$ (the latter is equivalently to large coupling between spectator and optical electrons) the line shape can be written as,

$$
\operatorname{Lim}_{\Gamma \rightarrow 0} I(x)=I_{o}(x)-\frac{3 \Gamma}{2 \pi} \frac{\left(x^{2}-1\right)}{\left[(x-1)^{2}+\Gamma^{2}\right]\left[(x+1)^{2}+9 \Gamma^{2}\right]}+\cdots
$$

and the effects from the interference terms vanish near line center.

For large $\Gamma$, either by $\gamma_{o}>>1$ or $\delta<<1$ (the latter is equivalently to small coupling between spectator and optical electrons),

$$
\operatorname{Lim}_{\Gamma \rightarrow \infty} I(x)=\frac{3}{4 \pi(2 x-1)^{2} \Gamma}+O\left(\frac{1}{\Gamma^{3}}\right)
$$

Therefore, $I(x)$ reduces to a narrow function with center at $x=x_{m}=1 / 2$. For large $\Gamma, I(x)$ can be approximated in the vicinity of the peak by [7]

$$
\tilde{I}(x) \approx \frac{\tilde{\Gamma} / \pi}{\left(x-x_{m}\right)^{2}+\tilde{\Gamma}^{2}}
$$

with

$$
\tilde{\Gamma}=\frac{3}{16 \Gamma}
$$


The analytic results show that for $\Gamma>>1$ the full calculation has a line shape with a single center at $x=x_{m}$ and an effective width inversely proportional to $\Gamma$. In addition, the interference terms reduce the far line wing intensity independent of the relative size of $\Gamma$.

\subsection{Numerical Results}

Results from Eqs. (3.6.1) and (3.6.4) are plotted in Fig. 2. The figure shows that for $\Gamma<<1$ the interference effects near the two line centers are small. The effects, however, are significant for $\Gamma \approx 1$ and dominate at line center for $\Gamma>>1$. The ratio of the profiles is plotted in Fig. 3 for $\Gamma \ll 1$ and $\Gamma \gg>1$, which are in agreement with the analytic results above. The large $\Gamma$ approximation in Eq. (3.7.5) is tested in Fig. 4 demonstrating that $\tilde{I}(\omega)$ is a reasonable approximation for $I(\omega)$ near line center. Note, however, that $\tilde{I}(\omega)$ has an incorrect $x^{-2}$ behavior at large detuning so always fails in the far wing.

\subsection{Quasi-static electric fields}

The quasi-static approximation assumes that the radiator finds itself in a statistical fluctuating potential. Typically, such theories compute the line shifts produced by the ion electric field followed by an average over the probability distribution of those fields. Rather than performing a complete calculation, the line shape is computed for small and large field values. In this manner, the effects of the interference terms can be more clearly ascertained.

The Stark effect is addressed in standard quantum mechanics textbooks so details are omitted. The problem consists of a radiator in an external, uniform static electric field, $\vec{F}$. The matrix elements of the external potential are given by [15]

$$
\left\langle J M|-\vec{d} \cdot \vec{F}| J^{\prime} M^{\prime}\right\rangle=-e a_{O} F(-1)^{J-M}\left(\begin{array}{ccc}
J & 1 & J^{\prime} \\
-M & 0 & M^{\prime}
\end{array}\right) S_{J J^{\prime}}^{1 / 2}
$$

where $F=|\vec{F}|$ and the field spatial direction was chosen along the z-axis.

In the large field limit, but still sufficiently small so there is no significant lower and upper level mixing, there are four distinct line centers at

$$
x=0,1 \text {, and } \pm \Phi
$$

where

$$
\Phi=\frac{2 e a_{o} R_{n s, n p}}{\sqrt{3} \hbar \delta} F
$$

and represents the Stark splitting scaled to the isolated radiator line separation energy. 
Line shape calculations are presented in Fig. 5 for small and large $\Phi$ values with and without the interference terms. The figure shows the narrowing effect of the interference terms similar to the zero field results as well as the $x^{-4}$ decay in the line wing, which explicitly occurs for each of the shifted components.

\subsection{Triplets, LS terms degeneracy, and nd spectator}

The calculations are repeated for the triplet states. This case has a total of $14 L S$ terms; that is, 4 and 10 terms in the lower and upper manifolds, respectively, leading to 432 line elements. Even though the triplet case yields larger matrices, making the same approximations (e.g.; neglect ion fields, degenerate $L S$-term structure, and same radial matrix elements for lower and upper configurations) lead to a numerically identical normalized profile as in the singlet example. For the triplet case, however, the total line strength is larger. That is, for the triplets

$$
\sum_{\mu \nu} \rho_{\mu} \vec{d}_{\mu v} \cdot \vec{d}_{v \mu}=12 \rho_{o} e^{2} a_{o}^{2} R_{1 s, 2 p}^{2}
$$

to be compared to Eq. (3.6.2).

For simplicity, all the calculations above assumed that the $L S$-term structure was degenerate. This approximation has been removed in Fig. 6 that compares calculations with degenerate and non-degenerate $L S$-term structure for the singlet levels. As before it is possible to see the individual lines without significant interference effects near line centers for $\Gamma<<1$. For $\Gamma \gg>1$, the interference effects also produce a sharp feature that, as expected from spreading the level energies, is shifted as well as slightly broader relative to the degenerate example. As in the degenerate case, the $x^{-2}$ term vanishes in the far wing.

The results so far have been limited to spectator electrons in the $n s$ and $n p$ sub-shells. It is straightforward to add radiative transitions between the configurations 1 snd and 2 pnd. The electron collisions now couple the $n p$ and $n d$ orbital tripling the width of lines with $n p$ as the spectator electron. These calculations require the radial dipole matrix element connecting the $n p$ and $n d$ sub-shells. Hydrogenic wavefunctions can be assumed for large $n$ giving [22]

$$
R_{n \ell, n \ell-1}=\frac{3 n}{2} \sqrt{n^{2}-\ell^{2}}
$$

or

$$
R_{n p, n d}=R_{n s, n p}+O\left(\frac{1}{n^{2}}\right) \approx R_{n s, n p}
$$


Calculations with and without the $n d$ orbital are presented in Fig. 7. The two line shapes are similar except for a relative shift produced by the additional line (here centered at $\omega_{o}-3 \delta / 2$ ) and a larger width due to the increased number of states available to the spectator electron.

\section{Spectator electron relaxation: $J K$ coupling}

The present work concentrates on excited spectator electron effects on the line shape of transitions involving tightly bound electrons. The $L S$ coupling scheme, however, is symmetric and not adequate for such asymmetric conditions. For these configurations the total energy is only weakly dependent on the spin of the excited electron and pair coupling is more suitable. The more common type is $J K$ coupling [15] (also called $j \ell$ coupling [7]), which for $n_{1} \ell_{1}$ and $n_{2} \ell_{2}$ the tightly and excited bound electrons quantum numbers, respectively, is defined by

$$
\vec{\ell}_{1}+\vec{s}_{1}=\vec{J}_{1}, \quad \vec{J}_{1}+\vec{\ell}_{2}=\vec{K}, \quad \vec{K}+\vec{s}_{2}=\vec{J}
$$

or $\left|\left\{\left[\left(\ell_{1}, s_{1}\right) J_{1}, \ell_{2}\right] K, s_{2}\right\} J m\right\rangle$. This example repeats the calculations in Section 3 using the same electron configurations as in Fig. 1, except that the angular momentum coupling scheme is changed from $L S$ to $J K$. All other approximations of Section 3 are retained.

Evaluation of the profile now involves $20 \mathrm{JK}$-terms; 6 in the lower level manifold and 14 in the upper level manifold generating a total of 768 line elements. The resolvent operator has 6 blocks contributing to the line shape. There are 3 blocks corresponding to upper levels with $J_{1}=1 / 2$ of size $(42 \times 42)$ for $\Delta m=0$ and $2(34 \times 34)$ for $\Delta m= \pm 1$. The other 3 blocks have upper levels with $J_{1}=3 / 2$ of size (68x68) for $\Delta m=0$ and 2(60x60) for $\Delta m= \pm 1$.

Formulas for the reduced dipole matrix elements are provided in Appendix C. Straightforward substitution of those results yields for the widths in Eq. (2.2.3),

$$
\gamma[2 p n s \rightarrow 1 s n s]=3 \gamma_{o} \quad \text { and } \quad \gamma[2 p n p \rightarrow 1 s n p]=\gamma_{o}
$$

where the notation denotes all terms involving the specified configurations and $\gamma_{o}$ in Eq. (3.3.2). The two angular momentum coupling schemes give identical level widths. This is expected since the sums in Eq. (2.2.3) are conserved under similarity transformations. [15]

The explicit dipole matrix elements will not be given here, however, note that the line strength for the two sets of diagonal blocks are 


$$
\begin{aligned}
& \left.\sum_{\mu \nu} \rho_{\mu} \vec{d}_{\mu \nu} \cdot \vec{d}_{v \mu}=\frac{16}{3} \rho_{o} e^{2} a_{o}^{2} R_{1 s, 2 p}^{2} \quad \text { [upper levels with } J_{1}=1 / 2\right] \\
& \left.\sum_{\mu \nu} \rho_{\mu} \vec{d}_{\mu \nu} \cdot \vec{d}_{v \mu}=\frac{32}{3} \rho_{o} e^{2} a_{o}^{2} R_{1 s, 2 p}^{2} \quad \text { [upper levels with } J_{1}=3 / 2\right]
\end{aligned}
$$

giving the same total line strength as in $L S$ coupling of Eqs. (3.6.2) plus (3.10.1). [15]

Numerical evaluation of the normalized profiles for the upper levels $J_{1}=1 / 2$ and $J_{1}=3 / 2$ blocks yield identical results. Furthermore, the normalized profiles exactly reproduced the $L S$ coupling numerical results of Section 3 including the vanishing of the $x^{-2}$ in the far line wing. As shown in Section 6, the far wing behavior is more general and associated with the separation of the radiator into tightly and excited bound electrons rather than specific to these examples.

\section{Optical and spectator electron relaxation}

The previous examples demonstrated the interference term effects on the line shape. In those examples the relaxation process was entirely associated with the spectator electron. Now relaxation processes are included for both the optical and spectator electron. The level structure is displayed in Fig. 8 that is similar to Fig. 1 but with two more configurations. Only singlet states in the $L S$ coupling scheme are considered. The approximations involving the radial integrals and level energies in the earlier examples are retained and Stark fields are neglected.

\subsection{Diagonal blocks}

Evaluation of the profile in this example involves 64 line elements with 3 similar (9x9) diagonal blocks that contribute to the profile; all others have zero dipole transitions. The block with $\Delta m=0$ involves the line elements,

$$
\{\mu v\}=3_{o} 1_{o}, 4_{o} 2_{o}, 5_{+} 2_{+}, 5_{-} 2_{-}, 6_{+} 2_{+}, 6{ }_{o} 2_{o}, 6 \_2_{-}, 7{ }_{o} 2_{o} \text {, and } 8_{o} 1_{o}
$$

where the level identification follows Fig. 8. The other two blocks have $\Delta m= \pm 1$ and give identical contributions to the profile so are not explicitly discussed.

\subsection{Reduced dipole elements}

The reduced dipole matrix element formulas in Section 3.2 still apply and the additional ones necessary for the width calculations are 


$$
\begin{aligned}
& S_{37}^{1 / 2}=-S_{73}^{1 / 2}=-S_{58}^{1 / 2}=-S_{85}^{1 / 2}=\xi \\
& S_{48}^{1 / 2}=-S_{84}^{1 / 2}=\frac{\xi}{\sqrt{3}} \\
& S_{68}^{1 / 2}=-S_{86}^{1 / 2}=\xi \sqrt{\frac{5}{3}} \\
& S_{78}^{1 / 2}=-S_{87}^{1 / 2}=-1,
\end{aligned}
$$

There are no new reduced dipole matrix elements for radiative transitions.

\subsection{Line width}

Straightforward substitution yields for the widths in Eq. (2.2.3), which are modified by the additional broadening due to levels 7 and 8 in Fig. 8,

$$
\begin{aligned}
& \gamma_{31}=\left(3+\frac{\xi^{2}}{2}\right) \gamma_{o} \\
& \gamma_{42}=\gamma_{52}=\gamma_{62}=\left(1+\frac{\xi^{2}}{2}\right) \gamma_{O}
\end{aligned}
$$

The additional level widths (although no radiative transitions are involved, they still appear in the resolvent) are given by

$$
\gamma_{81}=\gamma_{72}=\left(2+\frac{3 \xi^{2}}{2}\right) \gamma_{o}
$$

Here, the definition of $\gamma_{o}$ in Eq. (3.3.2) is retained. The additional interference terms for the $\Delta m=0$ block are 


$$
\begin{aligned}
& \tau_{3_{o} 1_{o}, 7_{o} 2_{o}}=\gamma_{o} \xi \\
& \tau_{4_{o} 2_{o}, 8_{o} 1_{o}}=-\frac{\gamma_{o} \xi}{\sqrt{3}}, \\
& \tau_{5_{+} 2_{+}, 8_{o} 1_{o}}=-\tau_{5_{-} 2_{-}, 8_{o} 1_{o}}=\tau_{6_{+} 2_{+}, 8_{o} 1_{o}}=\tau_{6_{-} 2_{-}, 8_{o} 1_{o}}=\frac{\gamma_{o} \xi}{\sqrt{2}} \\
& \tau_{6_{o} 2_{o}, 8_{o} 1_{o}}=-\gamma_{o} \xi \sqrt{\frac{2}{3}}, \\
& \tau_{7_{o} 2_{o}, 8_{o} 1_{o}}=\gamma_{o},
\end{aligned}
$$

Symmetry completes the matrix elements required for this block.

\subsection{Dipole line strength and resolvent operator}

The dot product of the dipole operators is identical to that in Eq. (3.4.1) except that two additional rows and columns of zeros are added to make a 9x9 matrix. The expressions for the $\Delta m=0$ block of $R^{-1}$ is given by the symmetric matrix

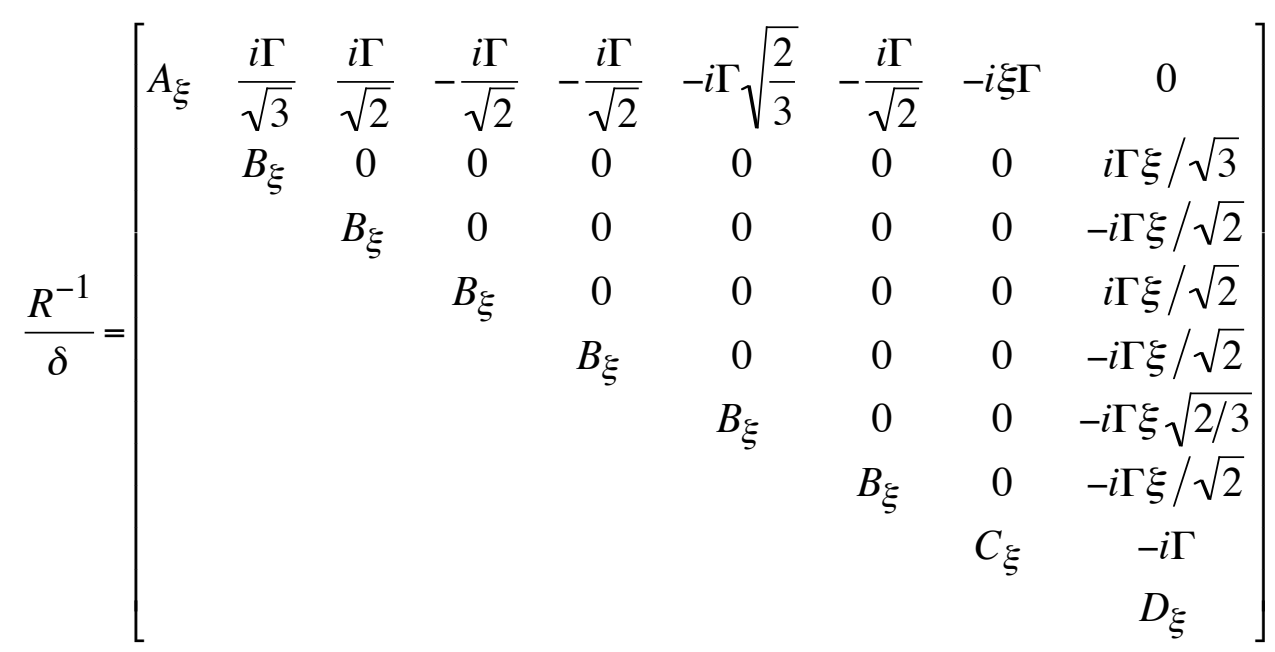

Again for brevity the lower triangle is left blank and the arguments of diagonal terms were suppressed,

$$
A_{\xi}(x, \Gamma)=x+1+i \Gamma\left(3+\frac{\xi^{2}}{2}\right)
$$




$$
\begin{aligned}
& B_{\xi}(x, \Gamma)=x-1+i \Gamma\left(1+\frac{\xi^{2}}{2}\right) \\
& C_{\xi}(x, \Gamma)=x+x_{7}+i \Gamma\left(2+\frac{3 \xi^{2}}{2}\right) \\
& D_{\xi}(x, \Gamma)=x+x_{8}+i \Gamma\left(2+\frac{3 \xi^{2}}{2}\right)
\end{aligned}
$$

where $x_{7}$ and $x_{8}$ are the scaled relative frequencies of levels 7 and 8.

\subsection{Line shape function}

The line shape function in Eq. (2.1) can now be obtained. Assuming that all initial states have the same occupation $\left(\rho_{\mu}=\rho_{O}\right.$ for all $\mu$ ), Eq, (2.1) yields identical contributions from the three (9x9) diagonal blocks of $R$. In addition, it was assumed that $x_{8}=-x_{7}=1$ simplifying the algebra. Although analytical results are possible for the line shape $I(x ; \xi)$, the expression is lengthy and not explicitly given. The line shape without interference terms is given by

$$
I_{o}(x ; \xi)=\frac{\Gamma}{2 \pi}\left\{\frac{6+\xi^{2}}{4(x+1)^{2}+\left(6+\xi^{2}\right)^{2} \Gamma^{2}}+\frac{3\left(2+\xi^{2}\right)}{4(x-1)^{2}+\left(2+\xi^{2}\right)^{2} \Gamma^{2}}\right\}
$$

where $I_{o}(\omega ; \xi)$ is normalized by the total line strength. As in the previous case, it is given by the sum of two Lorentz profiles described by the sum of level widths for the upper and lower states, which now includes relaxation processes from both the optical and spectator electrons.

\subsection{Analytical Limits}

Features of the line shape functions are obtained by considering various limits. Clearly, if the relaxation processes of the optical electron vanish, then

$$
\operatorname{Lim}_{\xi \rightarrow 0}\left\{\begin{array}{c}
I(x ; \xi) \\
I_{o}(x ; \xi)
\end{array}\right\}=\left\{\begin{array}{c}
I(x) \\
I_{o}(x)
\end{array}\right\}
$$

where $I(x)$ and $I_{o}(x)$ are the line shape with and without interference terms in Section 3.

In the far wing the profiles are given by (still assuming that $x_{8}=-x_{7}=1$ )

$$
\operatorname{Lim}_{x \rightarrow \infty} I(x ; \xi)=\frac{\xi^{2} \Gamma}{2 \pi x^{2}}\left\{1+\frac{1}{x}+O\left(\frac{1}{x^{2}}\right)\right\}
$$




$$
\operatorname{Lim}_{x \rightarrow \infty} I_{o}(x ; \xi)=\frac{\left(3+\xi^{2}\right) \Gamma}{2 \pi x^{2}}\left\{1+\frac{\xi^{2}}{\left(3+\xi^{2}\right) x}+O\left(\frac{1}{x^{2}}\right)\right\}
$$

Thus, the interference terms again lead to a reduced far wing independent of the value of $\Gamma$. In this case, however, the $x^{-2}$ term is present in both line shapes. That is, the interference terms remove the contribution to the $x^{-2}$ term in the far wing due to the relaxation processes associated with the spectator electron but not that associated with the optical electron.

For small $\Gamma$, it is possible to write

$$
\operatorname{Lim}_{\Gamma \rightarrow 0} I(x ; \xi)=I_{o}(x ; \xi)-\frac{\Gamma}{\pi} \frac{24\left(x^{2}-1\right)}{\left[4(x-1)^{2}+\Gamma^{2}\left(2+\xi^{2}\right)^{2}\right]\left[4(x+1)^{2}+\Gamma^{2}\left(6+\xi^{2}\right)^{2}\right]}+\cdots
$$

and the interference effects vanish near the line centers. Finally, $I(x ; \xi)$ does not reduce to a $\delta$ function for large $\Gamma$ as in the $\xi=0$ example.

\subsection{Numerical results}

Results for $I(x ; \xi), I_{o}(x ; \xi)$, and $I(x)$ are presented in Fig. 9. The calculations were performed for $x_{8}=-x_{7}=1$ and $\xi=1 / 6$ (approximate hydrogen scaling in Eq. (5.2.2) for $n=5$ ). The figure shows behavior similar to the previous example as $\Gamma$ varies from small to large values. In this second example, however, there is a $x^{-2}$ far wing behavior associated with the relaxation of the optical electron as well as less narrowing near line center with increasing $\Gamma$. That is, near line center the larger the relative value of $\xi$, the less apparent is the impact of the interference terms.

It is interesting to compare with a line shape that only includes the relaxation processes of the optical electron,

$$
I_{C}(x ; \xi)=\frac{\xi^{2} \Gamma}{2 \pi}\left\{\frac{1}{4(x+1)^{2}+\xi^{4} \Gamma^{2}}+\frac{3}{4(x-1)^{2}+\xi^{4} \Gamma^{2}}\right\}
$$

that has no interference terms and is the sum of two Lorentz profiles. This profile has a large frequency limit such that

$$
\operatorname{Lim}_{x \rightarrow \infty}\left\{\frac{I(x ; \xi)}{I_{C}(x ; \xi)}\right\}=\left\{1+\frac{12-\xi^{2} \Gamma^{2}\left(2+5 \xi^{2}\right)}{2 \xi^{2} x^{2}}+O\left(\frac{1}{x^{3}}\right)\right\}
$$


so that the two profiles have identical far wing limits.

Comparisons of $I(x ; \xi)$ and $I_{C}(x ; \xi)$ are presented in Fig. 10 showing that neglecting the broadening from the spectator electron is a good approximation in the far wing, as expected from Eq. (5.7.2). Near line center, however, neglecting the spectator electron-broadening can either under or over estimate the width depending on the degree of line overlapping.

A approximation to $I(x ; \xi)$ is given by

$$
P(x)=\frac{3}{\pi}\left\{\frac{\Gamma_{1}+\left(\Gamma_{2}-\Gamma_{1}\right) x^{2}}{\left(x^{2}-1\right)^{2}+4 \Gamma_{2}^{2}(2 x-1)^{2}}\right\}
$$

where

$$
\int_{-\infty}^{\infty} d x P(x)=1
$$

Note that for large detuning

$$
\operatorname{Lim}_{x \rightarrow \infty} P(x)=\frac{3\left(\Gamma_{2}-\Gamma_{1}\right)}{\pi x^{2}}+O\left(\frac{1}{x^{4}}\right)
$$

Thus, by setting

$$
\Gamma_{2}=\Gamma_{1}+\frac{\xi^{2}}{6} \Gamma
$$

yields the same far wing limit as $I(x ; \xi)$. Although $\Gamma_{1}$ is a free parameter, the line centers is well approximated by choosing $\Gamma_{1}=\Gamma$ as shown in Fig. 10. The profile $P(x)$ is in excellent agreement with $I(x ; \xi)$ for $\Gamma \leq 1$. For $\Gamma>1$, however, there is significant discrepancy away from line center until the profiles are dominated by the $x^{-2}$ term in the far wings.

\section{Far line wing limit}

The examples above yielded analytical profiles and explicitly showed that the $\Delta \omega^{-2}$ contribution from the spectator electron relaxation vanishes in the far wing. The vanishing of this term is a more general result and applies whenever the relaxation is due to an electron that is weakly coupled to the optically active electron. The following derivation helps clarify the conditions under which spectator electron contributions to the $\Delta \omega^{-2}$ far line wing vanish.

A formal expansion for large detuning of the line shape in Eq. (2.1) is given by (neglecting the quasi-static field) [4] 


$$
\begin{aligned}
\operatorname{Lim}_{\Delta \omega \rightarrow \infty} I(\omega) & =-\pi^{-1} \operatorname{Tr}\left\{\vec{d} \cdot \frac{1}{\Delta \omega}\left[1+\operatorname{Im} H(\omega) \frac{1}{\Delta \omega}+\cdots\right] \rho \vec{d}\right\} \\
& =-\pi^{-1} \sum_{\mu v} \sum_{\mu^{\prime} v^{\prime}} \rho_{\mu} \frac{1}{\Delta \omega_{\mu \nu}} \vec{d}_{\mu v} \cdot \vec{d}_{\mu^{\prime} v^{\prime}}^{*} \frac{1}{\Delta \omega_{\mu^{\prime} v^{\prime}}} \operatorname{Im} H_{\mu^{\prime} v^{\prime}, \mu v}(\omega)+\cdots
\end{aligned}
$$

and the leading term is of order $\Delta \omega^{-2}$.

To proceed, consider a radiator consisting of 'core' electrons plus a 'spectator' electron. [23] Now suppose that the spectral lines of interest involve transitions within the core states and the relaxation process only involves the spectator electron. If the spectator electron is assumed to have a small overlap with the core so that exchange can be neglected, then the total wavefunction can be written as simple products of core and spectator states. That is,

$$
|\mu\rangle \approx\left|\tilde{J}_{\mu} \tilde{M}_{\mu}\right\rangle\left|\sigma_{\mu} m_{\mu}\right\rangle
$$

Here, $\tilde{J}$ and $\tilde{M}$ are the total and $z$-component angular momentum for the core, respectively, with all other core quantum numbers suppressed for brevity. The spectator electron description is $\sigma=C n \ell j$ where $C$ indicates that its radial component also depends on the core configuration. The inner product of such wavefunctions is given by

$$
\begin{aligned}
\langle\mu \mid v\rangle & \approx\left\langle\tilde{J}_{\mu} \tilde{M}_{\mu} \mid \tilde{J}_{v} \tilde{M}_{v}\right\rangle\left\langle\sigma_{\mu} m_{\mu} \mid \sigma_{v} m_{v}\right\rangle \\
& =\delta_{\tilde{J}_{\mu} \tilde{J}_{v}} \delta_{\tilde{M}_{\mu} \tilde{M}_{v}} \delta_{j_{\mu} j_{v}} \delta_{\ell_{\mu} \ell_{v}} \delta_{m_{\mu} m_{v}}\left(\sigma_{\mu} \mid \sigma_{v}\right)
\end{aligned}
$$

where $\left(\sigma_{\mu} \mid \sigma_{v}\right)$ is the overlap integral of the spectator electron radial wavefunctions.

Typically, eigenfunctions of the total angular momentum squared are constructed using linear combinations of such product wavefunctions. [15] The leading term in Eq. (6.1) involves a trace; therefore, it is equivalent and also convenient to use the product wavefunctions in Eq. (6.2) rather than first couple the angular momentum using similarity transformations.

A direct consequence of the wavefunction approximation is to simplify the radiative transition dependence on the spectator angular momentum. Thus, the $q$-component of the dipole operator for the radiative transition involves

$$
\left\langle\tilde{J} \tilde{M}, \sigma m\left|r_{q}^{(1)}\right| \tilde{J}^{\prime} \tilde{M}^{\prime}, \sigma^{\prime} m^{\prime}\right\rangle=(-1)^{J-M}\left(\begin{array}{ccc}
\tilde{J} & 1 & \tilde{J}^{\prime} \\
-\tilde{M} & q & \tilde{M}^{\prime}
\end{array}\right) S_{\tilde{J} \tilde{J}^{\prime}}^{1 / 2} \delta_{j^{\prime} j} \delta_{l^{\prime} l} \delta_{m^{\prime} m} a_{o}\left(\sigma \mid \sigma^{\prime}\right)
$$

where $S_{\tilde{J} J^{\prime}}^{1 / 2}$ is the reduce dipole matrix element for the core alone. 
The collisional relaxation depends on the spectator electron as well as on the core through the detuning frequency and the spectator electron radial wavefunction. In the absence of magnetic fields, however, the detuning frequency is independent of magnetic quantum numbers,

$$
\Delta \omega_{\mu v}=\omega-\omega_{\tilde{J}_{\mu} \sigma_{\mu}}+\omega_{\tilde{J}_{v} \sigma_{v}}
$$

Furthermore, with the wavefunction approximation in Eq. (6.2), the width and shift operator reduces to

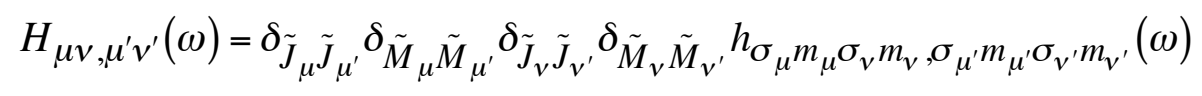

with all the dependence on the core angular momentum explicit in the kronecker's deltas and $h(\omega)$ the width and shift operator for the spectator electron only.

Substituting Eqs. (6.4) through (6.6) into the second line of Eq. (6.1), then the dot product of the dipole matrix elements can be summed over core magnetic quantum numbers. Assuming $\omega$ far from any of the overlapping lines centers (i.e.; $\Delta \omega_{\mu v} \rightarrow \Delta \omega$ for all $\mu$ and $v$ ) yields for the $\Delta \omega^{-2}$ coefficient,

$$
\sum_{\mu v} \sum_{\mu^{\prime} v^{\prime}} \vec{d}_{\mu \nu} \cdot \vec{d}_{\mu^{\prime} v^{\prime}}^{*} H_{\mu^{\prime} v^{\prime}, \mu v}(\omega) \propto \sum_{\tilde{J}} \sum_{\tilde{J}^{\prime}}\left|S_{\tilde{J} \tilde{J}^{\prime}}^{1 / 2}\right|^{2} \sum_{n \ell j m}^{r e s} \sum_{n^{\prime} \ell^{\prime} j^{\prime} m^{\prime}}^{r e s} \rho_{\tilde{J}}\left(\sigma \mid \sigma^{\prime}\right)^{2} h_{\sigma m \sigma m, \sigma^{\prime} m^{\prime} \sigma^{\prime} m^{\prime}}(\omega)+\cdots
$$

where the initial state populations are also assumed independent of magnetic quantum numbers. Note that the spectator state sums in Eq. (6.7) are restricted to states satisfying the factorization of the wavefunction and all other contributions denoted by the ellipsis.

The second-order theory and dipole approximation for the width and shift operator is discussed in Appendix B. Using the expressions for the dipole matrix elements yields

$$
\begin{gathered}
\sum_{m m^{\prime}} \sum_{\sigma m \sigma m, \sigma^{\prime} m^{\prime} \sigma^{\prime} m^{\prime}}(\omega) \propto\left|S_{\sigma^{\prime} \sigma}^{1 / 2}\right|^{2} g_{\sigma^{\prime}}^{\sigma}(\Delta \omega)\left\{1-\sum_{m m^{\prime} q}\left(\begin{array}{ccc}
j & 1 & j^{\prime} \\
-m & -q & m^{\prime}
\end{array}\right)^{2}\right\} \\
=\left|S_{\sigma^{\prime} \sigma}^{1 / 2}\right|^{2} g_{\sigma^{\prime}}^{\sigma}(\Delta \omega)\{1-1\}=0
\end{gathered}
$$

Thus, the $\Delta \omega^{-2}$ term vanishes. Here, $S_{\sigma \sigma^{\prime}}^{1 / 2}$ is the reduce dipole matrix element for the spectator electron and $g_{\sigma^{\prime}}^{\sigma}(\Delta \omega)$ is given by Eq. (2.2.4) except that the dependence on the spectator quantum numbers has been made explicit. The term in Eq. (6.8) vanishes level by level by summing over the spectator electron magnetic quantum numbers. Note that including the quasi- 
static field introduces a magnetic quantum number dependence on the detuning frequency. Nevertheless, this dependence can be neglected and the results in Eq. (6.8) remain valid if $\omega$ is far away from any Stark shifted line center.

Although the result in Eq. (6.8) is approximate (neglected exchange between core and spectator electrons) and may not exactly vanish in more complete calculations, the term should remain small relative to other contributions such as those from spectator states excluded from the restricted sum and the relaxation processes for the core.

\section{Aluminum dielectronic satellite lines}

Earlier work [27-32] investigated Stark broadening of the profiles and line-intensity ratios of dielectronic satellite lines. These studies, however, only included level widths neglecting the offdiagonal interference terms in the resolvent $R(\omega, F)$. For comparison, calculations for $\mathrm{Al}$ IV satellite lines are presented. The upper configurations in this example are $1 s 2 s^{2} 2 p^{6} 3 \ell$ with $\ell=s, p$, and $d$. The lower configurations are $1 s^{2} 2 s^{2} 2 p^{6}, 1 s^{2} 2 s^{2} 2 p^{5} 3 \ell$, and $1 s^{2} 2 s 2 p^{6} 3 \ell$. The radiative transitions of interest are

$$
1 s 2 s^{2} 2 p^{6} 3 \ell \rightarrow 1 s^{2} 2 s^{2} 2 p^{5} 3 \ell+\hbar \omega
$$

that appear as satellites to the resonant line in $\mathrm{Al} \mathrm{V}$,

$$
1 s 2 s^{2} 2 p^{6} \rightarrow 1 s^{2} 2 s^{2} 2 p^{5}+\hbar \omega .
$$

Collisional excitations or Stark mixing are not allowed between the initial and final states of the radiative transitions above.

The calculations include the electron collisional excitations between the $2 s$ and $2 p$ subshells as well as the collisional excitations and Stark mixing of the $3 \ell$ subshells. They also include the autoionization widths. $L S$ coupling is assumed and only the singlet states are considered. Note that the approximations in Sections 3 through 5 on term energies and radial dipole integrals are relaxed. Instead, the atomic data is obtained from a Dirac-Hartree-Slater self-consistent field code where the relativistic results are averaged to obtain the $L S$ representation. [33]

Area normalized profiles, which neglect the ion broadening, are plotted in Fig. 11 for plasma conditions expected in $K \alpha$ line emission experiments. [31] Interestingly, the interference effects at the higher densities lead to significant line narrowing. The effect of the ion microfields is displayed in Fig. 12 where profiles for several values of the Holtsmark field are plotted. Here, 


$$
F_{H}=\frac{F}{e / a_{e}^{2}}
$$

and

$$
\frac{4 \pi N_{e} a_{e}^{3}}{3}=1
$$

with $N_{e}$ the plasma free electron particle density. The plot shows that at $N_{e}=10^{22} \mathrm{~cm}^{-3}$ the ion microfield has a small effect on the broadening, which is dominated by electron impacts. At $N_{e}=10^{23} \mathrm{~cm}^{-3}$ the ion broadening becomes more important, but even large fields $\left(F_{H}=10\right)$ lead to a narrower spectral feature than neglecting the interference terms with $F_{H}=0$.

Not all dielectronic satellite lines will necessary display large corrections from the interference terms. In particular, if the quasi-static Stark fields dominate the line broadening, then they can mitigate their effects. As an example, at experimental plasma conditions the satellite lines of Ar XVI, which is a relatively ionized system and approximates linear Stark behavior, the interference effects were found to be in the order of a few percent. [19]

\section{Discussion}

The interference terms in electron impact broadening [4] were shown to be important in treating overlapping spectral lines resulting from the presence of excited spectator electrons. The schematic results from Sobelman [7,8] were reproduced with a concrete example involving twoelectron ions. An important consequence of the interference terms for cases where the relaxation process is completely due to the spectator electron is reducing the overlapping lines to a narrow spectral feature as the coupling between the spectator and optical electrons vanishes. That is, the overlapping lines coalesce and the relaxation processes of the spectator electrons no longer impacts the line shape. The equivalent effect happens for large plasma electron density and the line width becomes inversely proportional to the density.

The impact of the interference terms was also considered in the line wings. It was shown that the contribution to the leading $\Delta \omega^{-2}$ term in the far wing from the relaxation processes associated with the excited spectator electron vanishes. This outcome relied on negligible exchange effects between the core and spectator electrons in the radiator wavefunctions. The condition is common for configurations with a highly excited electron that experiences a small spin-dependent (exchange) Coulomb and spin-orbit interaction. [6,15,24] 
In the present examples the interference terms make a large impact on the line shape compared to their usually small effect on spectral lines used for plasma diagnostics. [3,5] The explanation follows from the width and shift operator, which shows that the interference terms involve the product of upper and lower state interactions with the perturbing electrons. For diagnostic lines lower-state broadening is often relatively small leading to small interference effects. [3] On the other hand, broadening due to an excited spectator electron is similar in both the lower and upper levels and the interference terms are no longer negligible.

For practical reasons the present results need to be distilled into a simple algorithm before they can be implemented in opacity codes. For opacity models where the profiles near line center have been compromised (e.g.; statistical line accounting methods $[25,26]$ ), the results suggest neglecting the highly excited spectator electron contributions in the width calculations.

Finally, earlier work [27-32] investigated Stark broadening profiles and line-intensity ratios of dielectronic satellite lines. These studies, however, only included level widths but neglected the interference terms. As shown here, the interference terms can modify the profiles of these overlapping dielectronic satellite lines introducing line narrowing and reduced wing intensities.

Acknowledgments: It is a pleasure to recognize valuable discussions with John I. Castor and Richard W. Lee. This work performed under the auspices of the U.S. Department of Energy by Lawrence Livermore National Laboratory under Contract DE-AC52-07NA27344. 


\section{APPENDIX A}

\section{Reduced dipole matrix elements}

Matrix elements of the radiator dipole operator are required for the line strength. In addition, both the quasi-static external potential and the width and shift operator are often treated in the dipole approximation. Consequently, the reduced dipole matrix elements play an important role in line shape calculations. [3,17-19]

Consider the internal radiator state $|J M\rangle$ where $J$ and $M$ are the total and the $z$-component angular momentum, respectively, where for brevity all other quantities specifying the state are suppressed. Then, by the Wigner-Eckart theorem [15]

$$
\begin{aligned}
\left\langle J M\left|r_{q}^{(1)}\right| J^{\prime} M^{\prime}\right\rangle & =(-1)^{J-M}\left(\begin{array}{ccc}
J & 1 & J^{\prime} \\
-M & q & M^{\prime}
\end{array}\right)\left\langle J\left\|r^{(1)}\right\| J^{\prime}\right\rangle \\
& =(-1)^{J-M}\left(\begin{array}{ccc}
J & 1 & J^{\prime} \\
-M & q & M^{\prime}
\end{array}\right) a_{o} S_{J J^{\prime}}^{1 / 2}
\end{aligned}
$$

defining the reduced dipole matrix element, $S_{J J^{\prime}}^{1 / 2}$, where $r_{q}^{(1)}$ is the $q^{\text {th }}$ spherical component of the radial vector, $\vec{r}$. The $S_{J J^{\prime}}^{1 / 2}$ contains the radial integrations and the dependence on quantum numbers other than those in the phase factor and 3-j symbol.

The reduced dipole matrix elements are not necessarily symmetric. Taking the complex conjugate of Eq. (A.1) yields

$$
(-1)^{q}\left\langle J^{\prime} M^{\prime}\left|r_{-q}^{(1)}\right| J M\right\rangle=(-1)^{J^{\prime}-M^{\prime}-q}\left(\begin{array}{ccc}
J^{\prime} & 1 & J \\
-M^{\prime} & -q & M
\end{array}\right) S_{J^{\prime} J}^{1 / 2} a_{o}
$$

The expressions in Eqs. (A.1) and (A.2) are real and consequently equal; thus, the symmetry properties of the $3-\mathrm{j}$ symbol yield

$$
S_{J^{\prime} J}^{1 / 2}=(-1)^{J-J^{\prime}} S_{J J^{\prime}}^{1 / 2}
$$

Note that the total dipole line strength is proportional to [15]

$$
(-1)^{J-J^{\prime}} S_{J J^{\prime}}^{1 / 2} S_{J^{\prime} J}^{1 / 2}=\left|S_{J J^{\prime}}^{1 / 2}\right|^{2} \geq 0
$$

Throughout the present work $S^{1 / 2}$ is in units of $a_{o}$ the Bohr radius. 


\section{APPENDIX B}

\section{Second-order width and shift operator}

A Liouville or double atom representation of the second-order width and shift operator is given by $[11,14]$

$$
\begin{aligned}
& H_{\alpha \beta, \alpha^{\prime} \beta^{\prime}}^{(2)}(\omega)=\frac{1}{\hbar^{2}} \int \frac{d \vec{k}}{(2 \pi)^{3}} \int_{-\infty}^{\infty} \frac{d \Omega}{2 \pi} S(k, \Omega)\left\{\sum _ { \sigma } \left[\delta_{\beta \beta^{\prime}} \frac{\tilde{V}_{\alpha \sigma}(\vec{k}) \tilde{V}_{\sigma \alpha^{\prime}}(-\vec{k}) f_{\sigma} f_{\alpha^{\prime}}^{-1}}{\Delta \omega_{\sigma \beta}-\Omega+i \eta}\right.\right. \\
& \left.\left.+\delta_{\alpha \alpha^{\prime}} \frac{\tilde{V}_{\beta^{\prime} \sigma}(-\vec{k}) \tilde{V}_{\sigma \beta}(\vec{k})}{\Delta \omega_{\alpha \sigma}-\Omega+i \eta}\right]-\tilde{V}_{\alpha \alpha^{\prime}}(\vec{k}) \tilde{V}_{\beta^{\prime} \beta}(-\vec{k})\left[\frac{1}{\Delta \omega_{\alpha^{\prime} \beta}-\Omega+i \eta}+\frac{f_{\alpha} f_{\alpha^{\prime}}^{-1}}{\Delta \omega_{\alpha \beta^{\prime}}-\Omega+i \eta}\right]\right\}
\end{aligned}
$$

where the sums are over all radiator internal states and $\eta \rightarrow 0^{+}$after the evaluation of the integrals. The radiator-electron interaction matrix elements are given by

$$
\tilde{V}_{\alpha \beta}(\vec{k})=\left\langle\alpha\left|\tilde{V}_{R}(\vec{k})\right| \beta\right\rangle
$$

where $\tilde{V}_{R}(\vec{k})$ is the Fourier transform of the radiator-electron interaction excluding the net Coulomb potential, $\hbar \vec{k}$ is the momentum transferred during the collision, and $S(k, \omega)$ the dynamic structure factor for an electron gas. The first 2 terms in Eq. (B.1) contribute to the level widths and the last 2 to the interference terms. The symbolic identity for real $\omega$

$$
\operatorname{Lim}_{\eta \rightarrow 0^{+}} \frac{1}{\omega \pm i \eta}=P \frac{1}{\omega} \mp i \pi \delta(\omega)
$$

where $P$ denotes the Cauchy principal part, shows that the line shift and width, $\operatorname{Re} H(\omega)$ and $\operatorname{Im} H(\omega)$, respectively, satisfy the dispersion relation

$$
\begin{aligned}
& \operatorname{Re} M(\omega)=\pi^{-1} P \int_{-\infty}^{\infty} d \omega^{\prime} \frac{\operatorname{Im} H\left(\omega^{\prime}\right)}{\omega-\omega^{\prime}} \\
& \operatorname{Im} M(\omega)=\pi^{-1} P \int_{-\infty}^{\infty} d \omega^{\prime} \frac{\operatorname{Re} H\left(\omega^{\prime}\right)}{\omega^{\prime}-\omega}
\end{aligned}
$$

Thus, the width and shift are not independent; knowledge of one implies the other.

\section{B.1 Dipole approximation}

The Fourier transform of the radiator-electron interaction is given by 


$$
\tilde{V}_{R}(\vec{k})=\frac{4 \pi e^{2}}{k^{2}} \sum_{a}\left[e^{i \vec{k} \cdot \vec{r}_{a}}-1\right]=\frac{4 \pi e^{2}}{k^{2}} 4 \pi \sum_{a \ell m} \sum_{\ell} i^{\ell}\left[j_{\ell}\left(k r_{a}\right)-\delta_{\ell 0}\right] Y_{\ell m}^{*}(\hat{k}) Y_{\ell m}\left(\hat{r}_{a}\right)
$$

where the sum $a$ is over all radiator bound electrons and the exponential was expanded in spherical harmonics with $j_{\ell}(x)$ the spherical Bessel function of order $\ell$. [34]

In most cases of interest $k^{-1}$ is much larger than the dimension of the radiator and the lowest order non-vanishing term leads to the dipole approximation, [3]

$$
\operatorname{Lim}_{k \rightarrow 0} \tilde{V}_{R}(\vec{k})=\tilde{v}_{R}(\vec{k})=\frac{4 \pi e^{2}}{k} \frac{4 \pi}{3} i \sum_{m} Y_{1 m}^{*}(\hat{k}) \sum_{a} r_{a} Y_{1 m}\left(\hat{r}_{a}\right)
$$

Assuming a single bound electron and noting that [15]

$$
r_{m}^{(1)}=\sqrt{\frac{4 \pi}{3}} r Y_{1 m}(\hat{r})
$$

yields

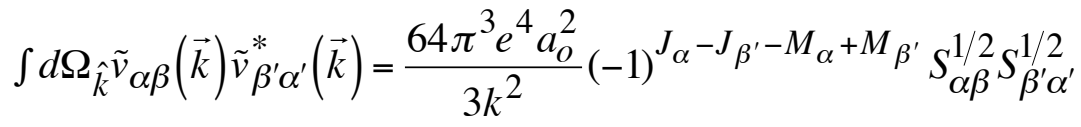

$$
\begin{aligned}
& \times \sum_{m}(-1)^{m}\left(\begin{array}{ccc}
J_{\alpha} & 1 & J_{\beta} \\
-M_{\alpha} & -m & M_{\beta}
\end{array}\right)\left(\begin{array}{ccc}
J_{\beta^{\prime}} & 1 & J_{\alpha^{\prime}} \\
-M_{\beta^{\prime}} & m & M_{\alpha^{\prime}}
\end{array}\right)
\end{aligned}
$$

From which follows, in combination with the orthogonality relations of 3-j symbols,

$$
\sum_{m_{\sigma}} \int d \Omega_{\hat{k}} \tilde{v}_{\alpha \sigma}(\vec{k}) \tilde{v}_{\sigma \alpha^{\prime}}^{*}(\vec{k})=\frac{64 \pi^{3} e^{4} a_{O}^{2}}{3 k^{2}} \frac{\left|S_{\alpha \sigma}^{1 / 2}\right|^{2}}{2 J_{\alpha}+1} \delta_{\alpha^{\prime} \alpha}
$$

Recall that $S_{\alpha \beta}^{1 / 2}$ is in units of $a_{o}$.

\section{B.2 Dynamic structure factor}

The dynamic structure factor for a classical, weakly coupled electron gas is given by [35]

$$
S(k, \omega)=\frac{S_{o}(k, \omega)}{|\varepsilon(k, \omega)|^{2}}
$$

with ideal gas structure factor and dielectric function

$$
S_{o}(k, \omega)=\frac{N_{e}}{\lambda_{e} k} \frac{\sqrt{2 \pi}}{\omega_{e}} \exp \left\{-\frac{1}{2 \lambda_{e}^{2} k^{2}} \frac{\omega^{2}}{\omega_{e}^{2}}\right\}
$$




$$
\varepsilon(k, \omega)=1+\frac{1}{k^{2} \lambda_{e}^{2}} W\left(\frac{1}{\lambda_{e} k} \frac{\omega}{\omega_{e}}\right)
$$

where

$$
W(z)=1-z \exp \left\{-\frac{z^{2}}{2}\right\} \int_{o}^{z} d y \exp \left\{y^{2} / 2\right\}+i \sqrt{\frac{\pi}{2}} \exp \left\{-\frac{z^{2}}{2}\right\}
$$

Here, $N_{e}, \lambda_{e}$, and $\omega_{e}$ are the electron number density, Debye length, and plasma frequency, respectively. The $W$-function has a small argument expansion and an asymptotic series, [35]

$$
\operatorname{Re} W(z)= \begin{cases}1-z^{2} \sum_{n} \frac{(-z)^{n}}{(2 n+1) ! !} & ,|z|<1 \\ -\sum_{n} \frac{(2 n-1) ! !}{z^{2 n}} & ,|z|>>1\end{cases}
$$

that are useful in approximating the momentum transfer integrals in $M^{(2)}$. [36]

\section{B.3 Integrals over momentum transfer}

The expression for the width involves an integral over the momentum transfer during the collision. In the second-order plus dipole approximation it has the form

$$
\int \frac{d \vec{k}}{(2 \pi)^{3}} S(k, \omega) \tilde{v}_{\alpha \beta}(\vec{k}) \tilde{v}_{\beta^{\prime} \alpha^{\prime}}^{*}(\vec{k}) \propto \int_{o}^{\infty} d k S(k, \omega)
$$

For large $k$,

$$
\operatorname{Lim}_{k \rightarrow \infty}\left\{\begin{array}{l}
|\varepsilon(k, \omega)|^{2} \rightarrow 1+O\left(k^{-2}\right) \\
S_{o}(k, \omega) \rightarrow \frac{N_{e}}{\lambda_{e} k} \frac{\sqrt{2 \pi}}{\omega_{e}}+O\left(k^{-2}\right)
\end{array}\right.
$$

and it follows from Eq. (B.2.1) that the integral in Eq. (B.3.1) diverges logarithmically. This is avoided by introducing a cutoff, $k_{m}$. [3,36] Fortunately, the resulting widths behave as

$$
\int_{o}^{\infty} d k S(k, \omega) \rightarrow \int_{o}^{k_{m}} d k S(k, \omega) \propto \log k_{m}
$$

and are only weakly dependent on the cutoff. 


\section{APPENDIX C}

\section{Reduced dipole matrix elements in LS and JK coupling}

The line profiles in the present wok require the reduced dipole matrix elements connecting the two-electron configurations,

$$
\begin{aligned}
& n_{1} \ell_{1} n_{2} \ell_{2} \leftrightarrow n_{1} \ell_{1} n_{2}^{\prime} \ell_{2}^{\prime} \\
& n_{1} \ell_{1} n_{2} \ell_{2} \leftrightarrow n_{1} \ell_{1}^{\prime} n_{2} \ell_{2}
\end{aligned}
$$

Here, results are provided in $L S$ and $J K$ coupling.

\section{C.1 LS coupling}

In $L S$ coupling the reduced dipole matrix element connecting the configurations in (C.1) is given by[15]

$$
\begin{array}{r}
\left\langle\left[\left(n_{1} \ell_{1}, n_{2} \ell_{2}\right) L, S\right] J\left\|\vec{r}_{2}\right\|\left[\left(n_{1} \ell_{1}, n_{2}^{\prime} \ell_{2}^{\prime}\right) L^{\prime}, S^{\prime}\right] J^{\prime}\right\rangle=-\delta_{S^{\prime} S}(-1)^{S+J^{\prime}+\ell_{1}+\ell_{2}^{>}}\left[J, J^{\prime}, L, L^{\prime}\right]^{1 / 2} \\
\times \sqrt{\ell_{2}^{>}}\left\{\begin{array}{ccc}
L & S & J \\
J^{\prime} & 1 & L^{\prime}
\end{array}\right\}\left\{\begin{array}{ccc}
\ell_{1} & \ell_{2} & L \\
1 & L^{\prime} & \ell_{2}^{\prime}
\end{array}\right\} a_{0} R_{n_{2} \ell_{2}, n_{2}^{\prime} \ell_{2}^{\prime}}
\end{array}
$$

where $[j]=2 j+1, \quad \ell_{2}^{>}=\max \left[\ell_{2}, \ell_{2}^{\prime}\right]$, and $R_{n \ell, n^{\prime} \ell^{\prime}}$ is the radial dipole integral. Similar manipulations yield for the configurations in (C.2) [15]

$$
\begin{aligned}
& \left\langle\left[\left(n_{1} \ell_{1}, n_{2} \ell_{2}\right) L, S\right] J\left\|\vec{r}_{1}\right\|\left[\left(n_{1}^{\prime} \ell_{1}^{\prime}, n_{2} \ell_{2}\right) L^{\prime}, S^{\prime}\right] J^{\prime}\right\rangle=-\delta_{S^{\prime} S}(-1)^{S+J^{\prime}+L+L^{\prime}+\ell_{2}+\ell_{1}^{>}} \\
& \times\left[J, J^{\prime}, L, L^{\prime}\right]^{1 / 2} \sqrt{\ell_{1}^{>}}\left\{\begin{array}{ccc}
L & S & J \\
J^{\prime} & 1 & L^{\prime}
\end{array}\right\}\left[\begin{array}{ccc}
\ell_{1} & \ell_{2} & L \\
L^{\prime} & 1 & \ell_{1}^{\prime}
\end{array}\right\} a_{O} R_{n_{1} \ell_{1}, n_{1}^{\prime} \ell_{1}^{\prime}}
\end{aligned}
$$

where $\ell_{1}^{>}=\max \left[\ell_{1}, \ell_{1}^{\prime}\right]$.

C.1 JK coupling

In $J K$ coupling the reduced dipole matrix element connecting the same two pair of configurations above are given by [15] 


$$
\begin{gathered}
\left\langle\left\{\left[\left(\ell_{1}, s_{1}\right) J_{1}, \ell_{2}\right] K, s_{2}\right\} J\left\|\vec{r}_{2}\right\|\left\{\left[\left(\ell_{1}, s_{1}\right) J_{1}, \ell_{2}^{\prime}\right] K^{\prime}, s_{2}\right\} J^{\prime}\right\rangle=-\delta_{J_{1}^{\prime} J_{1}}(-1)^{1 / 2+J^{\prime}-J_{1}+\ell_{2}} \\
\times\left[J, J^{\prime}, K, K^{\prime}\right]^{1 / 2} \sqrt{\ell_{2}^{>}}\left\{\begin{array}{ccc}
K & 1 / 2 & J \\
J^{\prime} & 1 & K^{\prime}
\end{array}\right\}\left\{\begin{array}{ccc}
J_{1} & \ell_{2} & K \\
1 & K^{\prime} & \ell_{2}^{\prime}
\end{array}\right\} a_{o} R_{n_{2} \ell_{2}, n_{2}^{\prime} \ell_{2}}
\end{gathered}
$$

and

$$
\begin{gathered}
\left\langle\left\{\left[\left(\ell_{1}, s_{1}\right) J_{1}, \ell_{2}\right] K, s_{2}\right\} J\left\|\vec{r}_{1}\right\|\left\{\left[\left(\ell_{1}^{\prime}, s_{1}\right) J_{1}, \ell_{2}\right] K^{\prime}, s_{2}\right\} J^{\prime}\right\rangle=\delta_{J_{1}^{\prime} J_{1}}(-1)^{K+K^{\prime}+J^{\prime}+J_{1}+J_{1}^{\prime}+\ell_{2}+\ell_{1}^{>}} \\
\times\left[J_{1}, J_{1}^{\prime}, K, K^{\prime} J, J^{\prime}\right]^{1 / 2} \sqrt{\ell_{1}^{>}}\left\{\begin{array}{ccc}
K & 1 / 2 & J \\
J^{\prime} & 1 & K^{\prime}
\end{array}\right\}\left\{\begin{array}{ccc}
J_{1} & \ell_{2} & K \\
K^{\prime} & 1 & J_{1}^{\prime}
\end{array}\right\}\left\{\begin{array}{ccc}
\ell_{1} & 1 / 2 & J_{1} \\
J_{1}^{\prime} & 1 & \ell_{1}^{\prime}
\end{array}\right\} a_{o} R_{n_{1} \ell_{1}, n_{1}^{\prime} \ell_{1}^{\prime}}
\end{gathered}
$$




\section{References}

[1] D. Mihalas, Stellar Atmospheres (Freeman \& Co., San Francisco, 1978)

[2] C.A. Iglesias et al, HEDP 5, 97(2009)

[3] H.R. Griem, Spectral Line Broadening in Plasmas (Academic Press, New York \& London, 1974)

[4] M. Baranger, Phys. Rev. 111, 494(1958)

[5] C.R. Vidal, J. Cooper \& E.W. Smith, Astrophys.J.Supp. 25, 37(1973)

[6] M.A. Gigoso et al, Astron. Astrophys. 466, 1189(2007)

[7] I.I. Sobelman, Excitation of Atoms and Broadening of Spectral Lines (Springer-Verlag, Berlin, 1980); V.I.

[8] Alekseev \& I.I. Sobelman, Sov.Phys. JETP 28, 991(1969)

[9] E.W. Smith \& C.F. Hooper, Phys.Rev. 157, 126(1966)

[10] E.W. Smith, Phys.Rev. 166, 102(1968)

[11] T. Hussey, J.W. Dufty \& C.F. Hooper, Phys.Rev. 12, 1084(1975)

[12] T. Hussey, J.W. Dufty \& C.F. Hooper, Phys.Rev. 16, 1248(1977)

[13] J.W. Dufty \& D.B. Boercker, JQSRT 16, 1065(1976)

[14] C.A. Iglesias, HEDP 1, 42(2005)

[15] R. Cowan, The Theory of Atomic Structure (California Press, Berkeley, 1974)

[16] M. Baranger, Phys. Rev. 112, 855(1958)

[17] L.A. Woltz \& C.F. Hooper, Phys.Rev. 38, 4766(1988)

[18] A. Calisti et al, Phys.Rev. A42, 5433(1990)

[19] P.A. Loboda et al, Laser \& Particle Phys. 18, 275(2000)

[20] C.A. Iglesias et al, Phys.Rev. A31, 1698(1985)

[21] An computationally fast version of the APEX program [20] with several new features is available, C.A. Iglesias et al, JQSRT 65, 303(2000)

[22] H.A. Bethe and E.E. Salpeter, Quantum Mechanics of One-and Two-electron Atoms (Plenum Publishing Co., N.Y., 1977)

[23] J.I. Castor suggested the separation of the radiator into a core plus a spectator electron and neglecting exchange effects; private communication.

[24] R.D. Cowan \& K.L. Andrew, J.Opt.Soc.Am. 55, 502(1965)

[25] J. Bauche, C. Bauche-Arnoult, \& M. Klapisch, Adv. Atom. Phys. 23, 131(1987) 
[26] A. Bar-Shalom et al., Phys. Rev. A40, 3183(1989)

[27] J.M. Bañon, M. Koenig \& H. Nguyen, J. Phys. B18, 4195(1985)

[28] J.M. Bañon \& H. Nguyen, J. Phys. B20, 2989(1987)

[29] J.M. Bañon, L.L. Sanchez-Soto \& E. Bernabeu, J. Phys. B22, 3382(1989)

[30] L.A. Woltz et al, Phys. Rev. A44, 1281(1991)

[31] J.J. MacFarlane et al, Phys. Rev. E47, 2748(1993)

[32] R.C. Mancini et al, Phys.Rev. E54, 4147(1996)

[33] F.P. Larkin, J. Phys. B2, 244(1970)

[34] M. Abramowitz \& I.A. Stegun, Handbook of Mathematical Functions, NBS, 1972

[35] S. Ichimaru, Basic Principles of Plasma Physics (Benjamin, Reading, 1973)

[36] R.W. Lee, JQSRT 40, 561(1988)

Possible References

[17a] R.C. Mancini et al, CPC 63, 314(1991) 


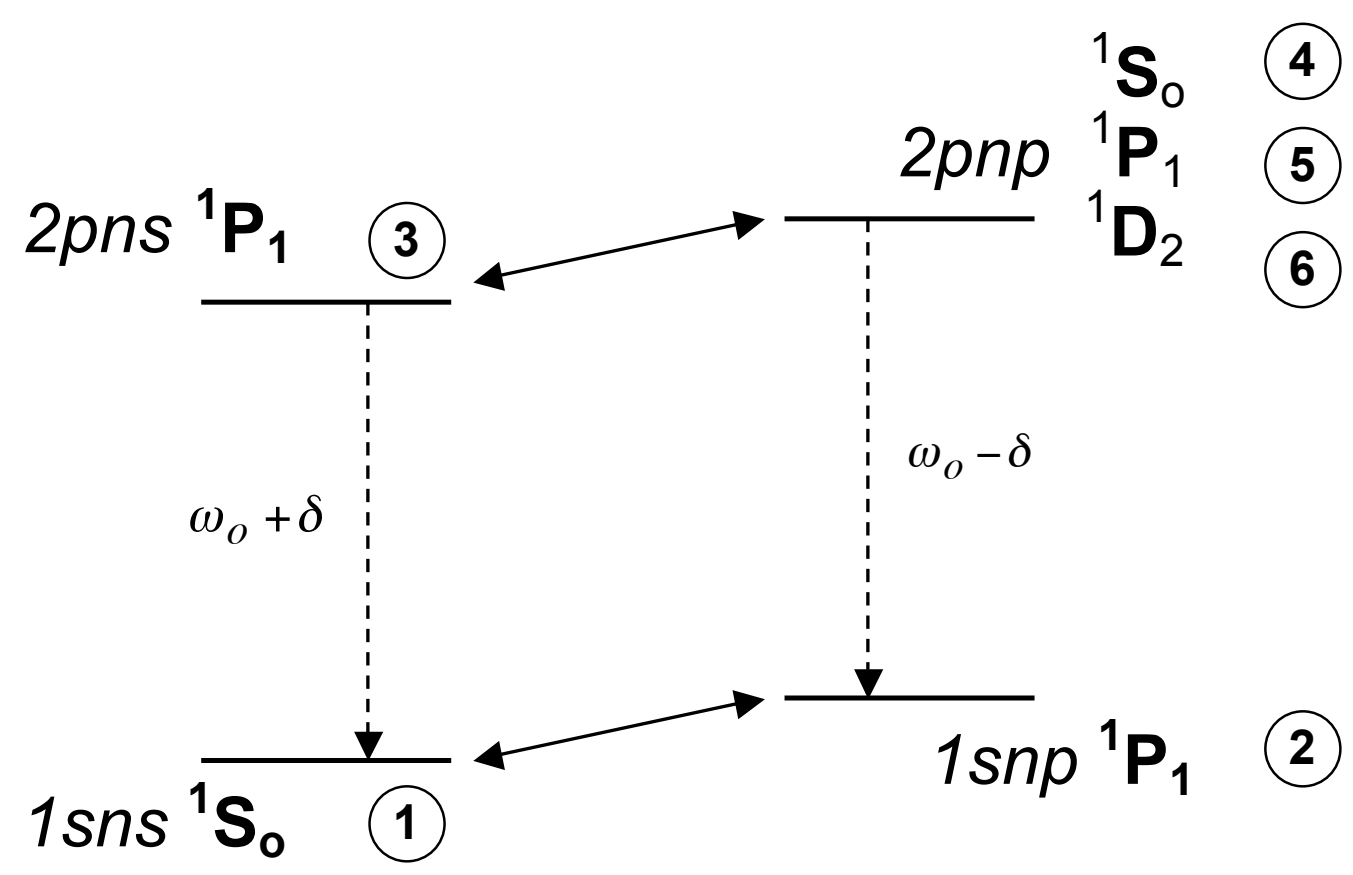

Fig. 1 Schematic energy diagram of singlet levels in two-electron configurations using $L S$ coupling. The circled numbers indicate level labels. The double-headed solid arrows denote allowed electron collisional excitations and Stark field mixing while the dashed arrows allowed radiative transitions. 

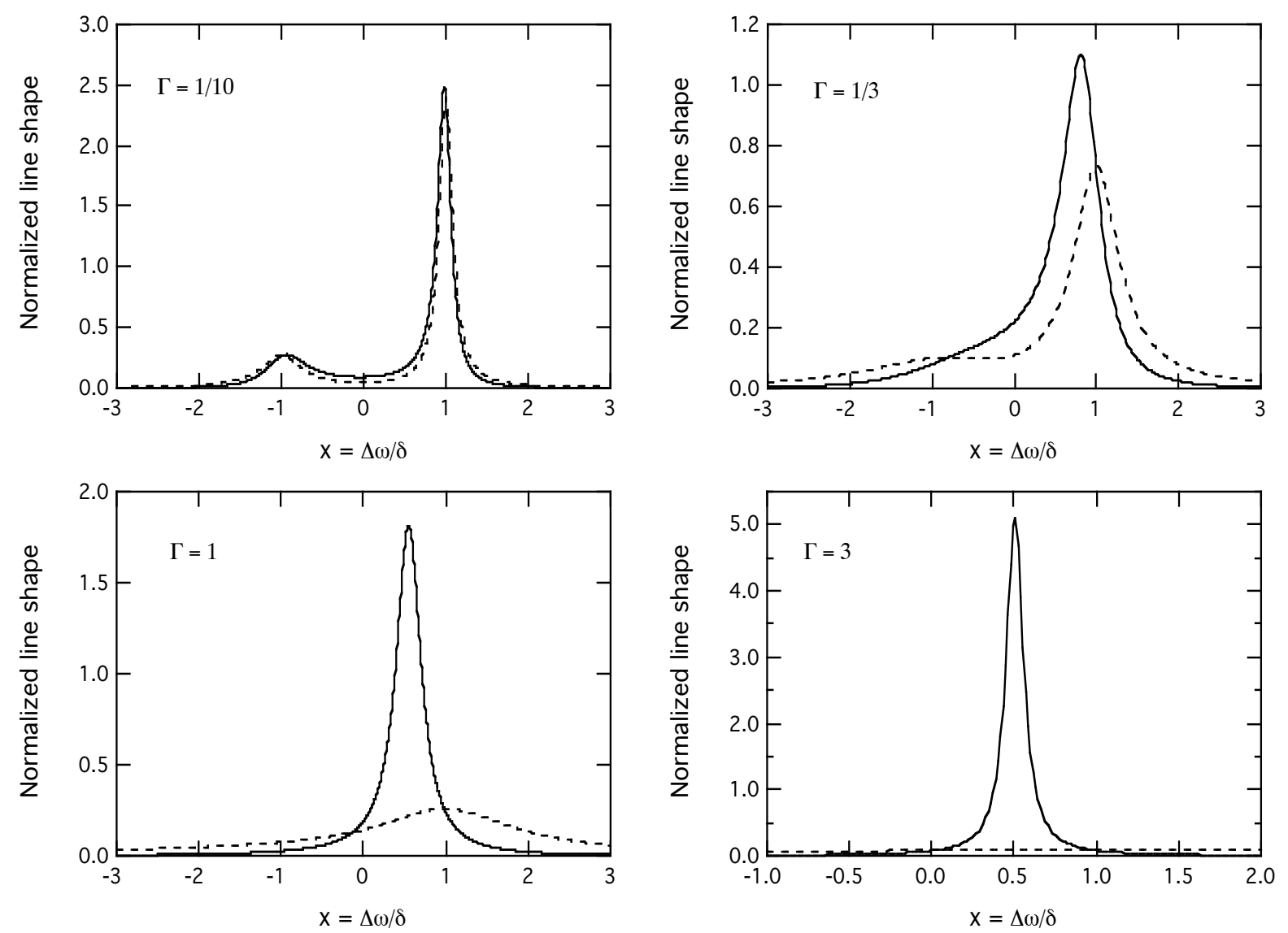

Fig. 2 Plots of $I(x)($ solid $)$ and $I_{o}(x)($ dash) as a function of detuning for $\Gamma=1 / 10,1 / 3,1$, and 3. 


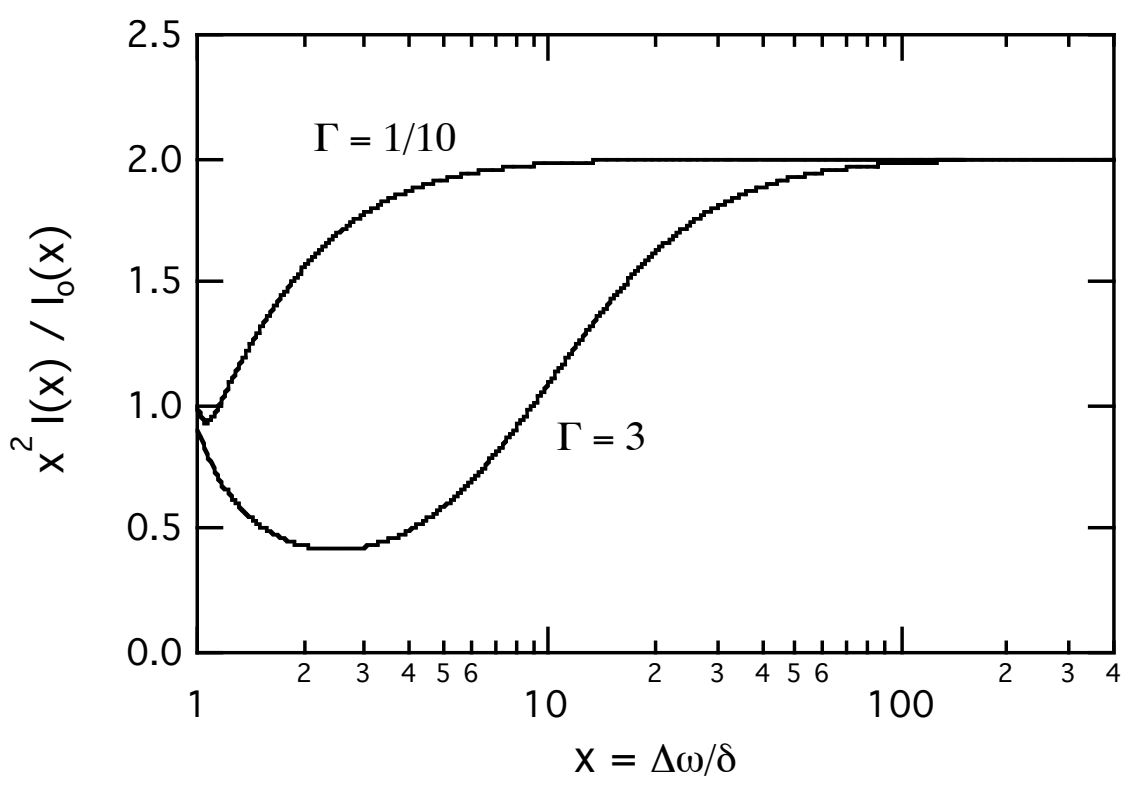

Fig. 3 The ratio $x^{2} I(x) / I_{o}(x)$ as a function of detuning for $\Gamma=1 / 10$ and 3 . 

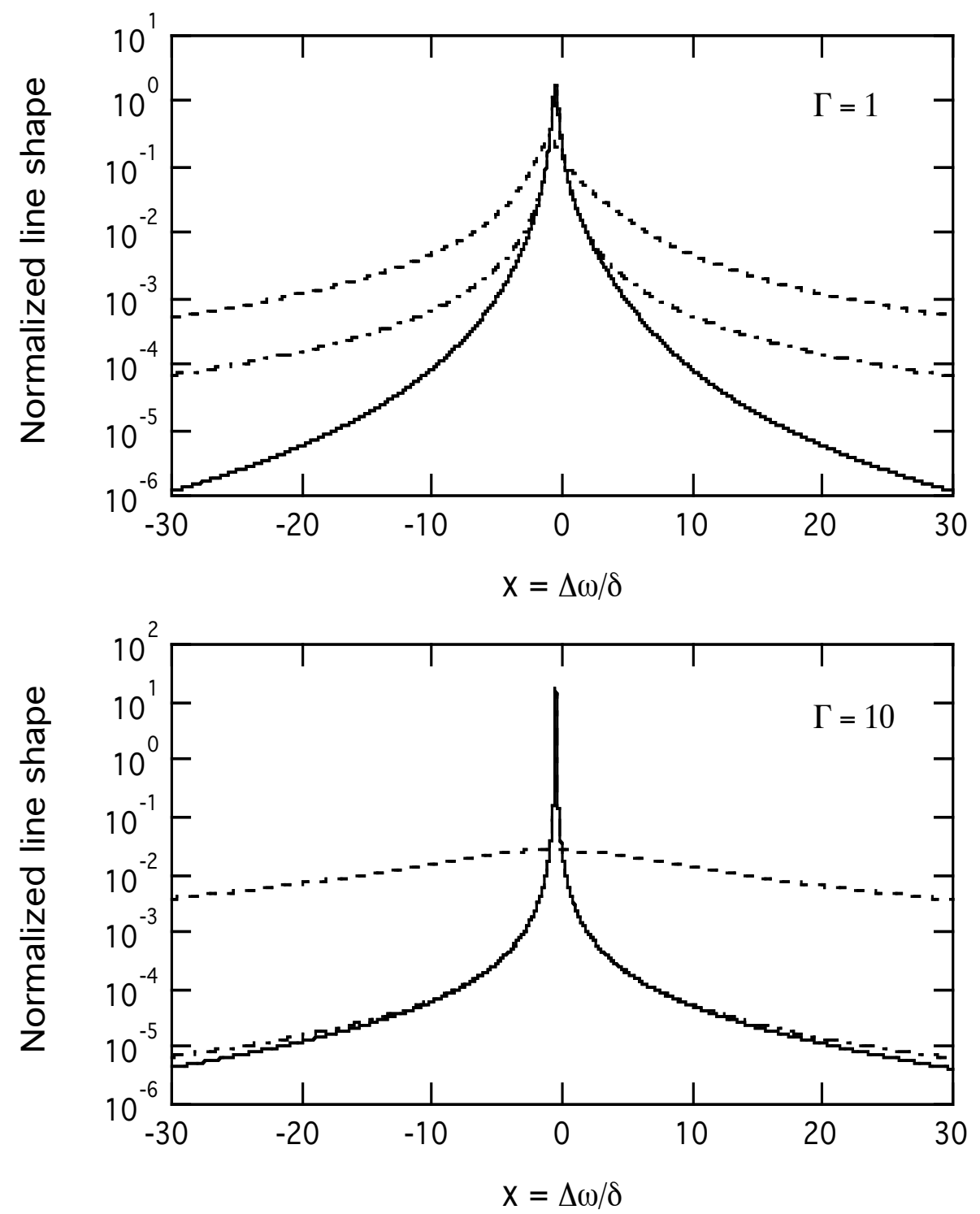

Fig. 4 Comparisons of $I(x)($ solid $), I_{o}(x)($ dash), and $\tilde{I}(x)($ dot-dash) as a function of detuning for $\Gamma=1$ and 10 . 

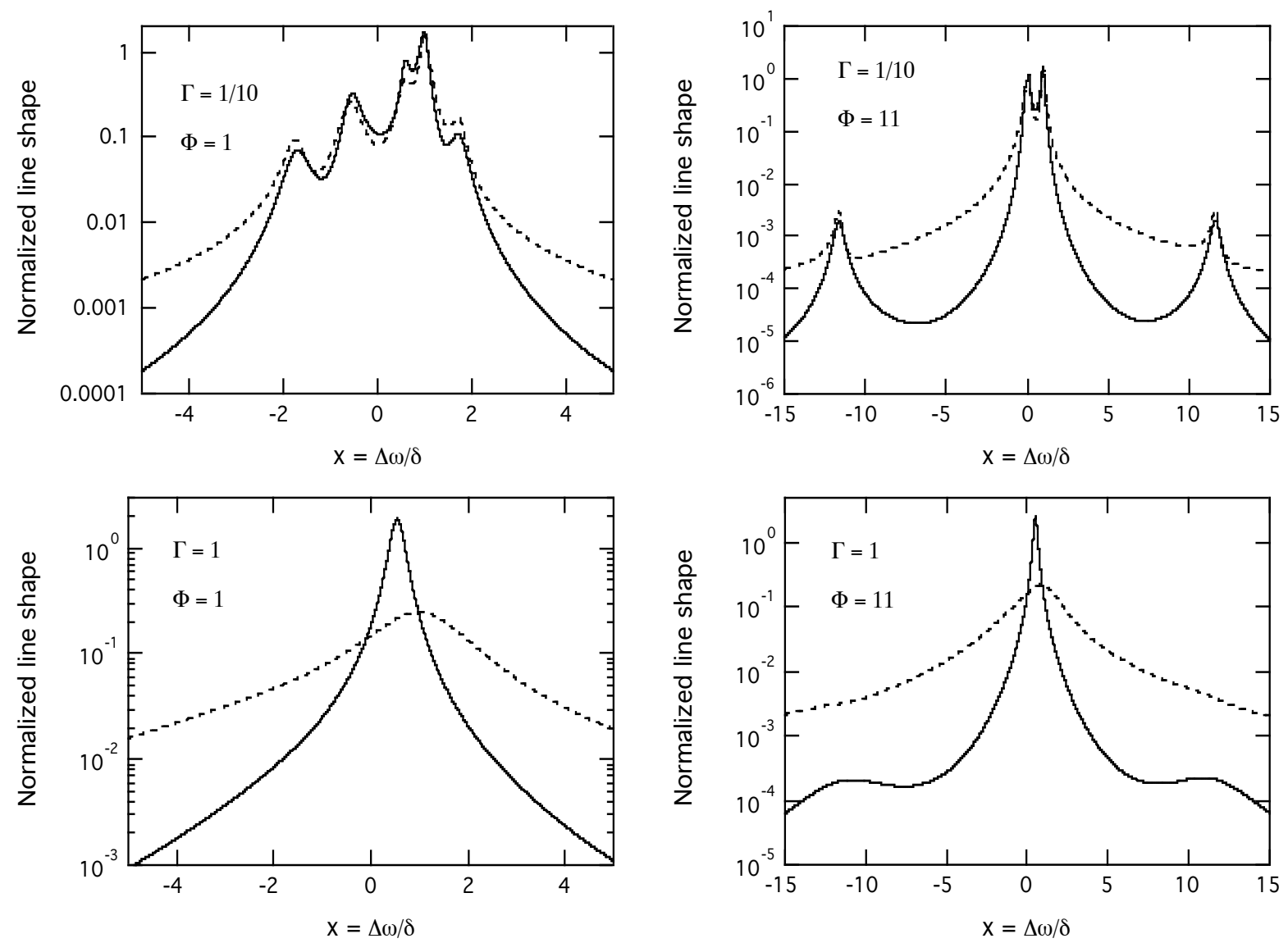

Fig. 5 Comparisons of line shapes at $\Gamma=1 / 10$ and 1 with (solid) and without (dash) interference terms as a function of detuning for small and large Stark fields, $\Phi=1$ and 11 . 

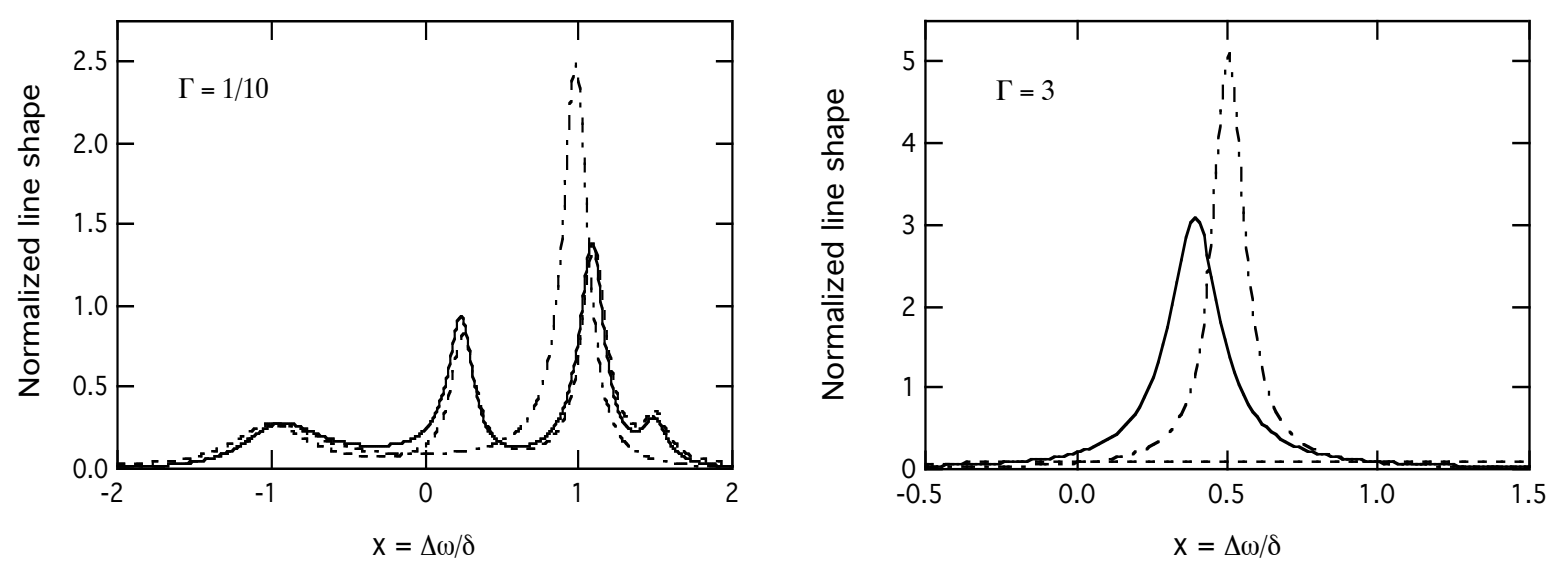

Fig. 6 Comparisons of $I(x)($ solid $)$ and $I_{o}(x)($ dash) with non-degenerate $L S$-term structure as a function of detuning for $\Gamma=1 / 10$ and 3 . Also included for reference is $I(x)$ with degenerate $L S$-term structure (dot-dash). 


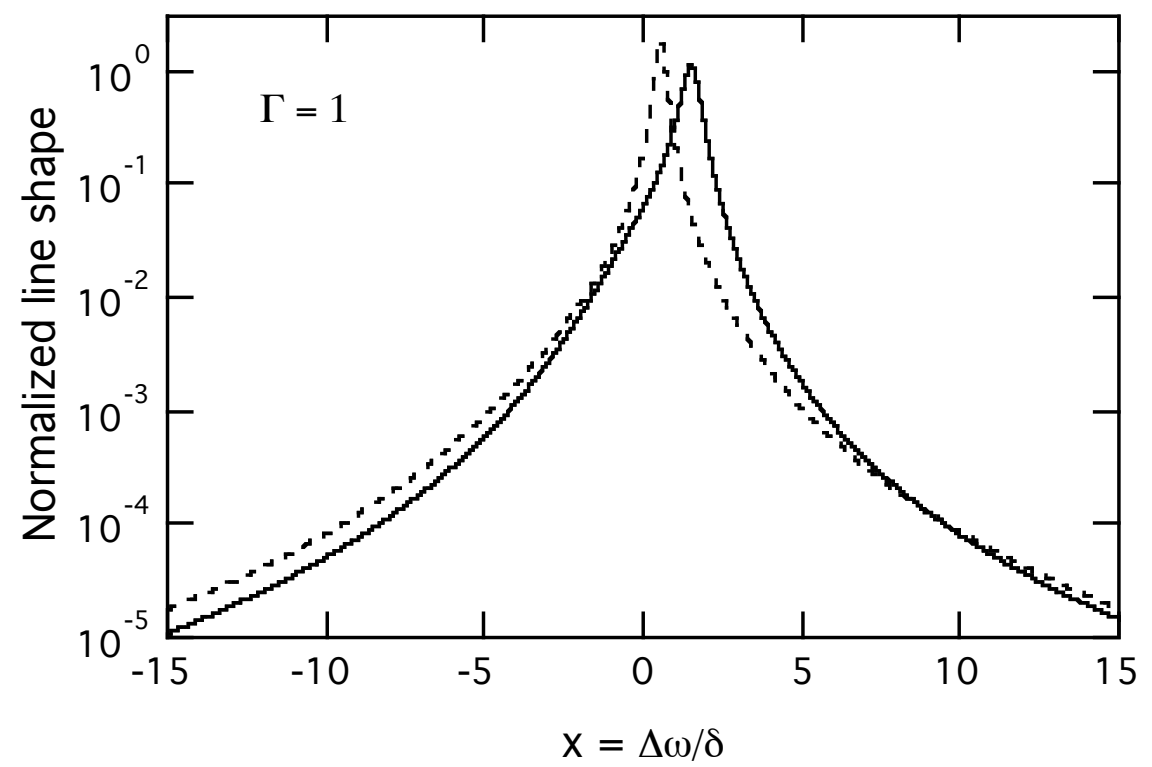

Fig. 7 Comparisons of line shapes as a function of detuning including interference terms for $\Gamma=1$ with $n s$ and $n p$ spectators (dash) and with $n s, n p$, and $n d$ spectators (solid) where both assumed degenerate $L S$-term structure. 


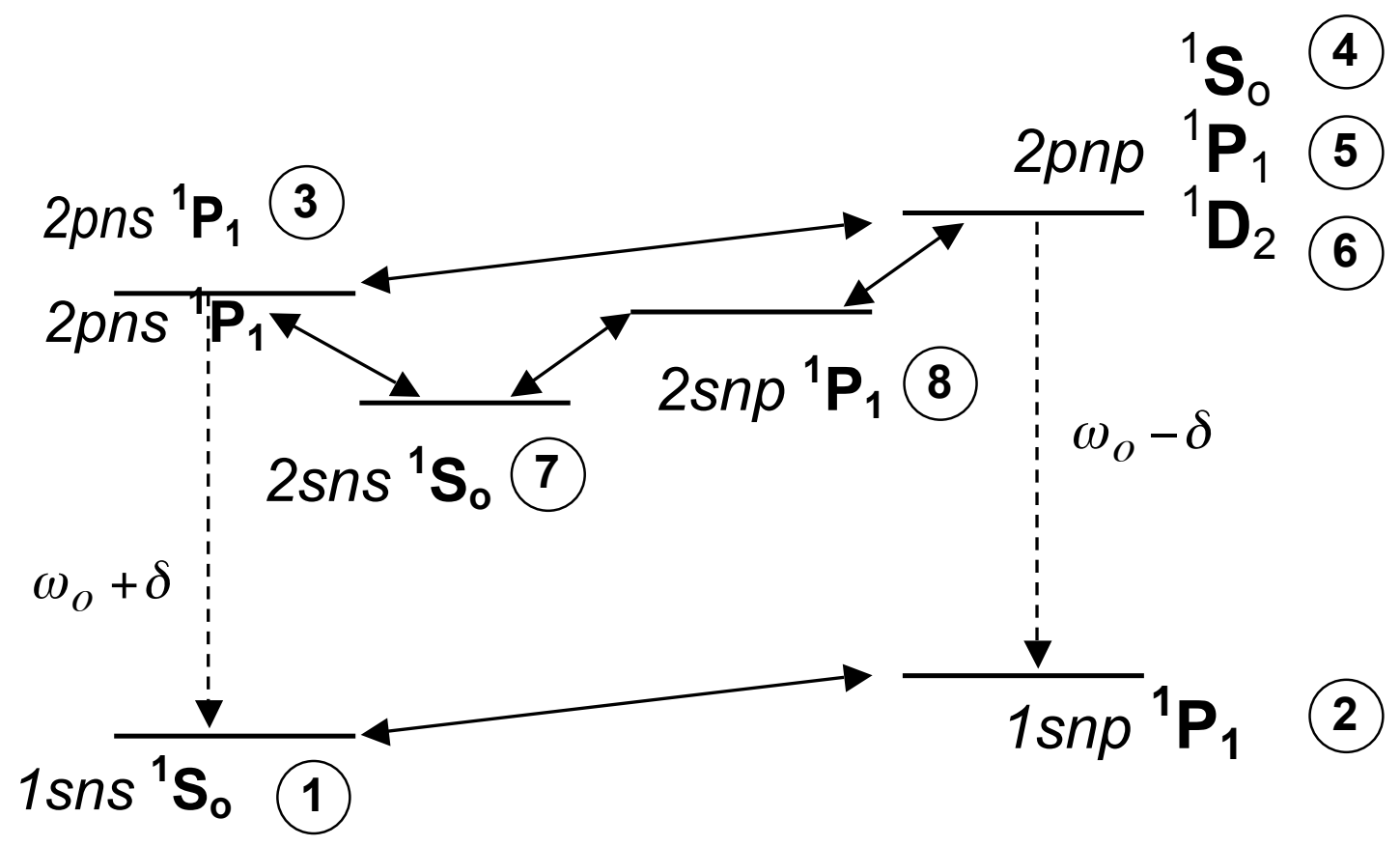

Fig. 8 Same as Fig. 1 plus two more configurations interacting with the upper level manifold. 

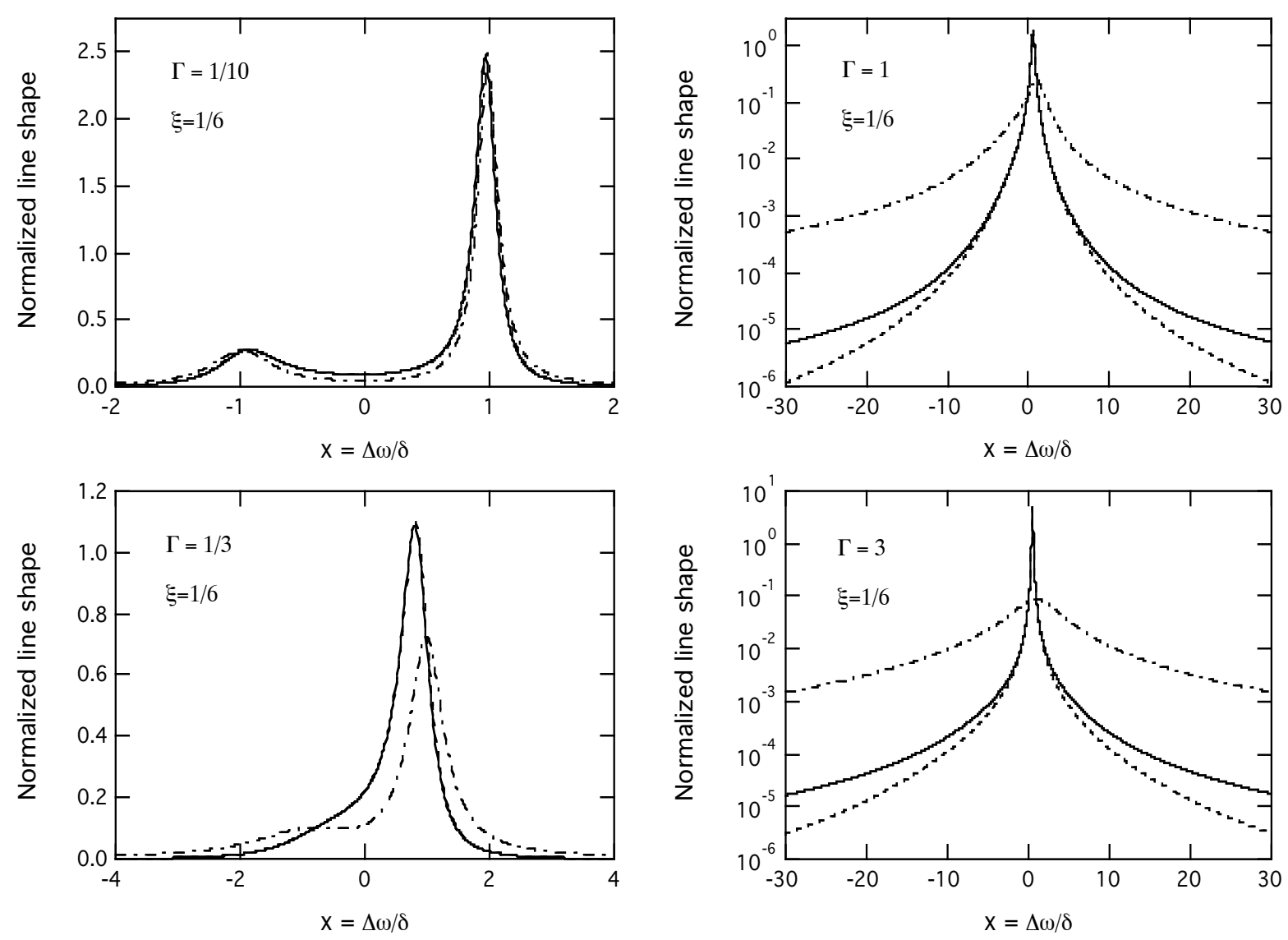

Fig. 9 Plots of $I(x ; \xi)$ (solid) and $I_{o}(x ; \xi)$ (dot-dash) with $\xi=1 / 6$ as a function of detuning for $\Gamma=1 / 10,1 / 3,1$, and 3. Also included are results for $I(x ; \xi=0)($ dash $)$. Note the logarithmic scale for $\Gamma=1$ and 3 . 

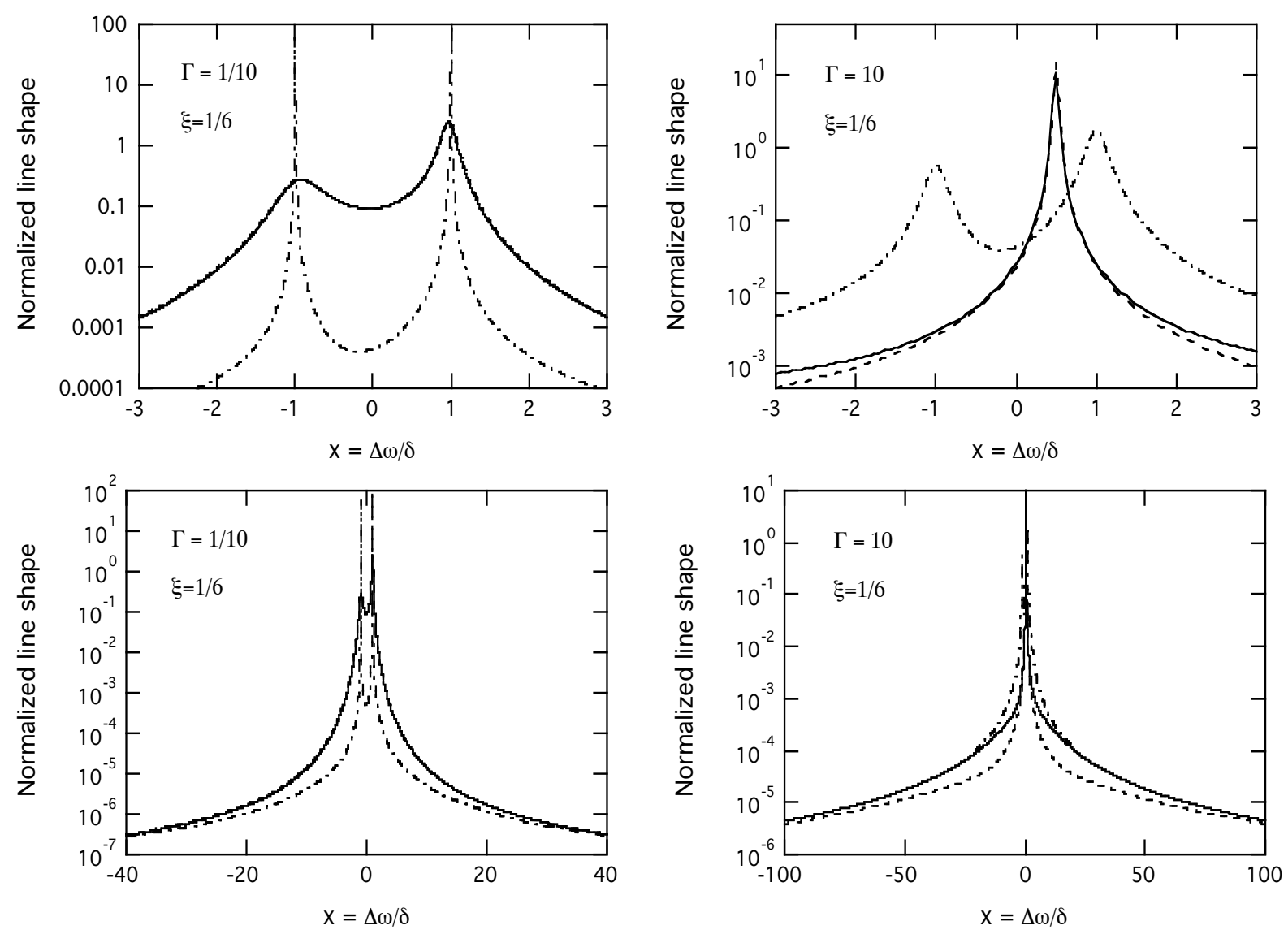

Fig. 10 Plots of $I(x ; \xi)$ (solid), $P(x)\left(\right.$ dash), and $I_{C}(x ; \xi)($ dot-dash) for $\xi=1 / 6$ as a function of detuning for $\Gamma=1 / 10$ and 10 . Linear and logarithmic plots emphasize line centers and wings, respectively. 

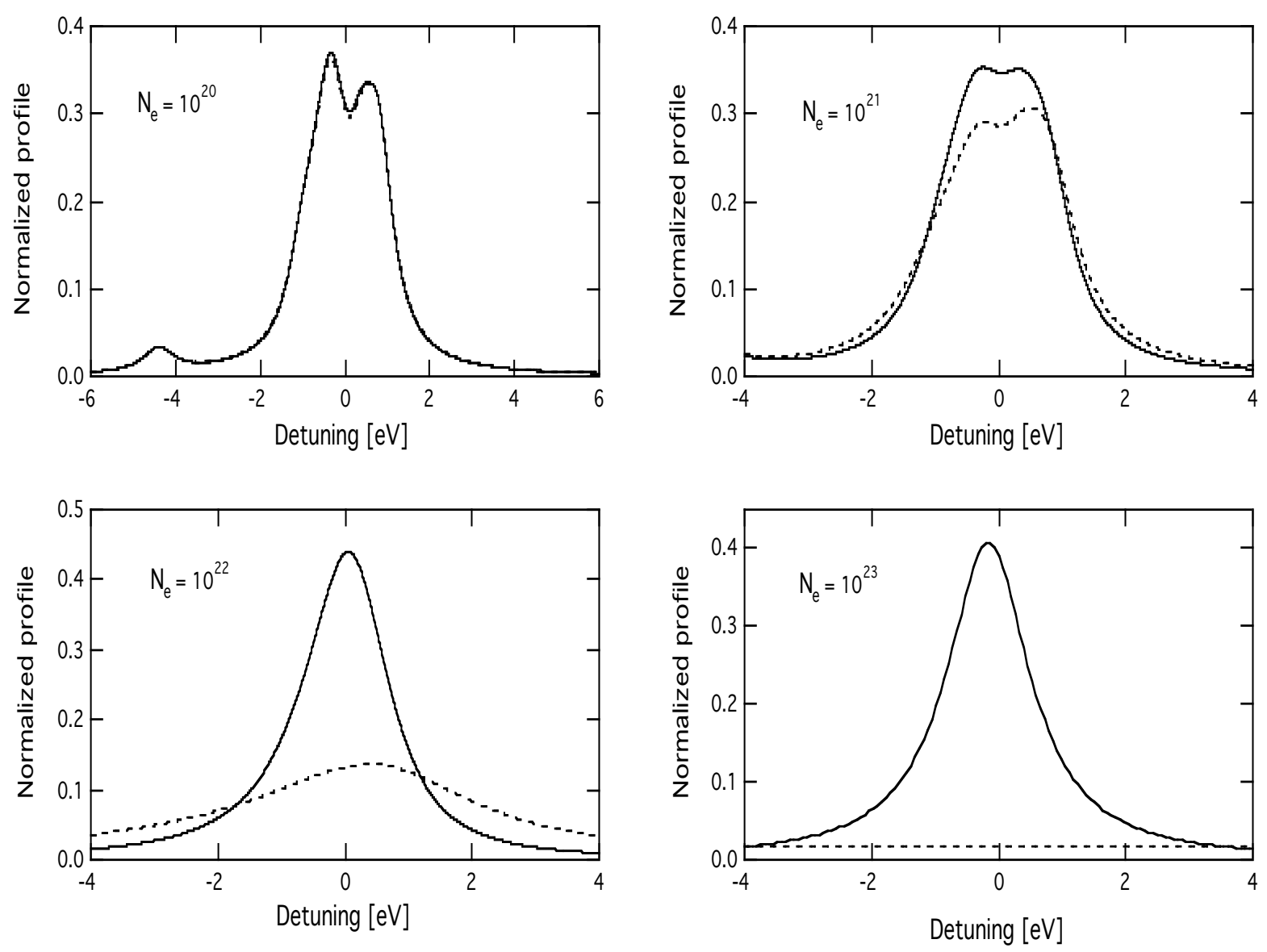

Fig. 11 Strength normalized profiles of Al IV $K \alpha$ satellite lines with a spectator electron in the $n=3$ shell with (solid) and without (dash) interference terms at $20 \mathrm{eV}$ temperature and free electron densities, $N_{e}=10^{20}, 10^{21}, 10^{22}$, and $10^{23} \mathrm{~cm}^{-3}$. The zero detuning was arbitrarily chosen near the peak of the profile with interference terms and ion broadening was neglected. 

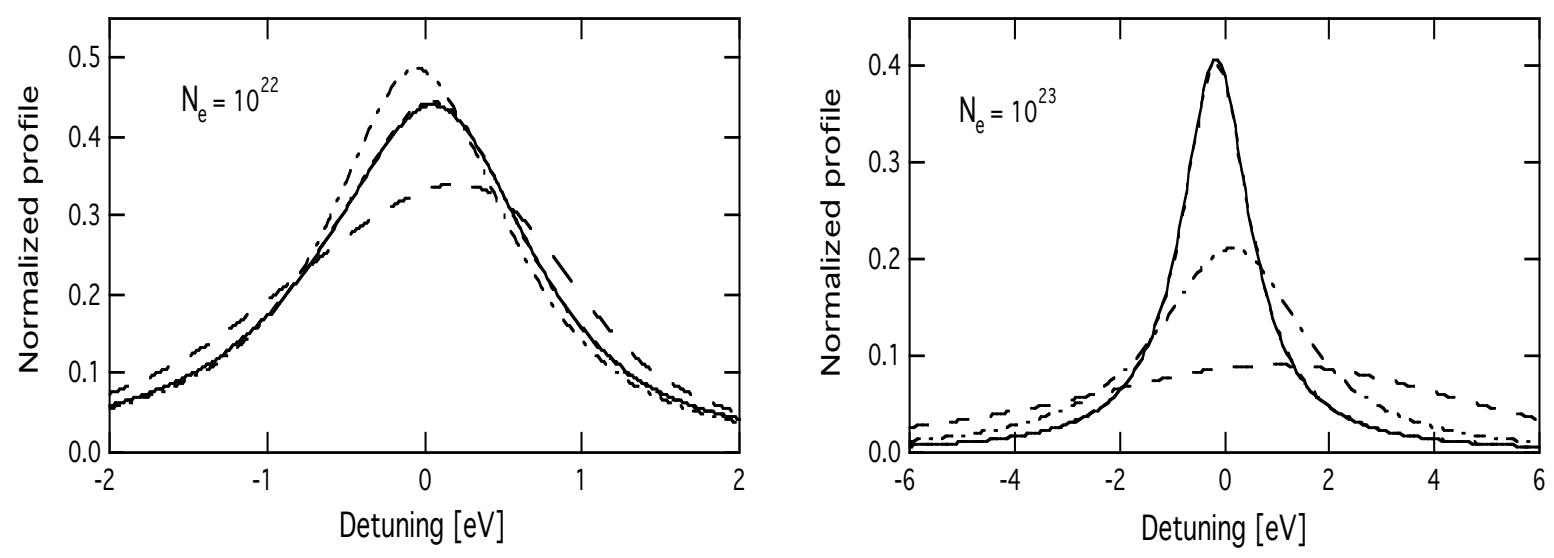

Fig. 12 Strength normalized profiles of Al IV $K \alpha$ satellite lines with a spectator electron in the $n=3$ shell with interference terms at $20 \mathrm{eV}$ temperature and $N_{e}=10^{22}$ and $10^{23} \mathrm{~cm}^{-3}$. The Holtsmark fields values are: $F_{H}=0$ (solid), $F_{H}=1$ (short dash) almost indistinguishable from zero field result, $F_{H}=5$ (dot-dash), and $F_{H}=10$ (long dash). 